TRANSACTIONS OF THE

AMERICAN MATHEMATICAL SOCIETY

Volume 361, Number 3, March 2009, Pages 1397-1427

S 0002-9947(08)04666-7

Article electronically published on October 24, 2008

\title{
COMPLETELY BOUNDED MAPPINGS AND SIMPLICIAL COMPLEX STRUCTURE IN THE PRIMITIVE IDEAL SPACE OF A $C^{*}$-ALGEBRA
}

\author{
ROBERT J. ARCHBOLD, DOUGLAS W. B. SOMERSET, AND RICHARD M. TIMONEY
}

\begin{abstract}
We consider the natural contraction from the central Haagerup tensor product of a $C^{*}$-algebra $A$ with itself to the space of completely bounded maps $C B(A)$ on $A$ and investigate those $A$ where there exists an inverse map with finite norm $L(A)$. We show that a stabilised version $L^{\prime}(A)=$ $\sup _{n} L\left(M_{n}(A)\right)$ depends only on the primitive ideal space $\operatorname{Prim}(A)$. The dependence is via simplicial complex structures (defined from primal intersections) on finite sets of primitive ideals that contain a Glimm ideal of $A$. Moreover $L^{\prime}(A)=L(A \otimes \mathcal{K}(H))$, with $\mathcal{K}(H)$ the compact operators, which requires us to develop the theory in the context of $C^{*}$-algebras that are not necessarily unital.
\end{abstract}

\section{INTRODUCTION}

In previous work [10] we characterised, via a condition on the ideal structure, those (unital) $C^{*}$-algebras $A$ where the canonical contraction $\theta_{Z}$ from the central Haagerup tensor product $A \otimes_{Z, h} A$ to the space $\mathrm{CB}(A)$ of completely bounded maps on $A$ is isometric. This completed earlier work of Somerset 22] which had shown sufficiency of the condition (all Glimm ideals of $A$ should be primal ideals). Further details on previous related work is given in [10].

Our aim here is to study those $A$ for which $\theta_{Z}$ is injective but not necessarily isometric. We recall that $\theta_{Z}$ is injective if and only if every Glimm ideal of $A$ is 2-primal (see [22, Corollary 6] and Theorem 3.8 (i) below). We then let $L(A)$ denote the norm of the inverse of $\theta_{Z}$, a constant associated with $A$ which can be $\infty$ (see Definition 4.1). It turns out that the value of $L(A)$ (or even its finiteness) cannot be determined by the ideal structure of $A$, but a stabilised version $L^{\prime}(A)=$ $\sup _{n} L\left(M_{n}(A)\right)$ can be determined via the combinatorics of the ideal structure of $A$ (Theorem 6.1). This is perhaps unexpected in view of the equivalences: $\theta_{Z}$ is isometric $\Longleftrightarrow L(A)=1 \Longleftrightarrow$ every Glimm ideal of $A$ is primal $\Longleftrightarrow L^{\prime}(A)=1$. In fact $L^{\prime}(A)$ depends only on the primitive ideal space $\operatorname{Prim}(A)$ (Theorem 6.2).

It is tempting to conjecture that $L^{\prime}(A)$ is $L(A \otimes \mathcal{K}(H)$ ) (for $\mathcal{K}(H)$ the compact operators on a separable infinite dimensional Hilbert space), and we do indeed prove this in Corollary 6.3. However, it brings us into the realm of non-unital algebras, and we need to develop a suitable notion of a central Haagerup tensor product

Received by the editors February 1, 2007.

2000 Mathematics Subject Classification. Primary 46L05, 47B47, 46L06, 46L07.

The work of the third author was supported in part by the Science Foundation Ireland under grant 05/RFP/MAT0033. 
$A \otimes_{Z, h} A$ in the non-unital case. We do this in section $\$ 3$ and work throughout in this generalised context. We remark that a more common approach of passing to the multiplier algebra $M(A)$ works if we express conditions in terms of the ideal structure of $M(A)$ ([10, Corollary 9]), but this can be very different to that of $A$ (see [10, Example 12] and Example 3.10 below).

The result about $L^{\prime}(A)$ being determined via the combinatorial structure of the ideals of $A$ (Theorem 6.1) proceeds by establishing comparisons with combinatorially defined models which we call simplicial-spoke algebras (introduced in Notation 4.6). They have one Glimm ideal $G_{\infty}$ that fails to be a primal ideal (all other Glimm ideals are in fact primitive ideals) and a finite number of primitive ideals containing $G_{\infty}$. Aside from this finiteness, the subcollections of the primitive ideals containing $G_{\infty}$ which have primal intersection can be quite general in these spoke algebras. We proceed by relating $L(A)$ and $L^{\prime}(A)$ (for arbitrary $A$ ) to these constants for simplicial-spoke algebras linked to $A$ (Theorems 5.1 and 6.1).

Due to the abstraction involved in these results, we provide some explicit examples (and inequalities) in \$7 including an example where $L(A)<\infty$ but $L^{\prime}(A)=\infty$. This example arises as a sum of a sequence of examples where $L(A)$ stays bounded, but $L^{\prime}(A)$ increases to infinity. Our computations lead us to consider seemingly elementary questions about matrix norms, and it may be of independent interest to generalise Proposition 7.5 (for example). We conclude with examples of nilpotent Lie groups $G$ where $L^{\prime}\left(C^{*}(G)\right)=L\left(C^{*}(G)\right)=\infty$ while $\theta_{Z}$ is injective for $A=C^{*}(G)$.

Throughout this work we make fundamental use of the tracial geometric mean 23 and its link with the Haagerup tensor norm (see Lemma 2.1).

We also use the following notation. For $A$ a $C^{*}$-algebra, $\operatorname{Primal}(A)$ is the set of primal ideals, Min-Primal $(A)$ the minimal primal ideals and $\operatorname{Glimm}(A)$ the Glimm ideals. Recall that an ideal $I \in \operatorname{Id}(A)$ is called $n$-primal if whenever $J_{1}, \ldots, J_{n} \in \operatorname{Id}(A)$ with $J_{1} J_{2} \cdots J_{n}=0$ there is $1 \leq i \leq n$ with $J_{i} \subseteq I$ [8]. An equivalent definition in terms of the topology of $\operatorname{Prim}(A)$ is that $I$ is $n$-primal when $P_{1}, P_{2}, \ldots, P_{n} \in \operatorname{Prim}(A / I)$ implies there exists a net $\left(P_{\alpha}\right)_{\alpha}$ in $\operatorname{Prim}(A)$ converging to all of $P_{1}, P_{2}, \ldots, P_{n}$. To say $I \in \operatorname{Primal}(A)$ means that $I$ is $n$-primal for each $n \geq 2$ 5. Recall that for a (not necessarily unital) $C^{*}$-algebra $A$, a Glimm ideal is the kernel of an equivalence class in $\operatorname{Prim}(A)$, where $P, P^{\prime} \in \operatorname{Prim}(A)$ are defined to be equivalent if $f(P)=f\left(P^{\prime}\right)$ for each $f \in C^{b}(\operatorname{Prim}(A))$. We equip $\operatorname{Glimm}(A)$ with the completely regular topology, so that it is the complete regularisation of $\operatorname{Prim}(A)$ [15.

We shall frequently use the well known fact that there is a homeomorphism $\operatorname{Prim}(A) \rightarrow \operatorname{Prim}\left(A \otimes M_{n}\right)$ (respectively $\left.\operatorname{Prim}(A) \rightarrow \operatorname{Prim}(A \otimes \mathcal{K}(H))\right)$, given by $P \mapsto P \otimes M_{n}$ (resp. $P \mapsto P \otimes \mathcal{K}(H)$ ). For the case of $A \otimes M_{n}$, this is elementary, and in the case of $A \otimes \mathcal{K}(H)$ it follows easily from (for example) [17, Theorems 5 and 6$]$ and the exactness of $\mathcal{K}(H)$. It is also elementary that the Glimm ideals of $A \otimes M_{n}=M_{n}(A)$ are those of the form $G \otimes M_{n}$ where $G \in \operatorname{Glimm}(A)$, and a similar remark applies to primal ideals.

We denote by $\mathbb{S}(A)$ the states of $A, \mathbb{P}(A)$ the pure states and $\mathbb{F}_{f}(A)$ the finite type I factorial states. Recall that $\mathbb{F}_{f}(A)$ can be described [1] as those $\phi \in \mathbb{S}(A)$ where the GNS representation $\pi_{\phi}: A \rightarrow \mathcal{B}\left(H_{\pi_{\phi}}\right)$ has commutant $\pi_{\phi}(A)^{\prime}$ a factor of finite type I. Equivalently $\phi$ is a convex combination $\sum_{i=1}^{n} t_{i} \psi_{i}$ where $\psi_{i} \in \mathbb{P}(A)$ $(1 \leq i \leq n)$ are equivalent pure states of $A$. 
For a tensor $u=\sum_{j=1}^{\ell} a_{j} \otimes b_{j}$ we use the (variation on standard) notation $u=\mathbf{a} \odot \mathbf{b}$, where $\mathbf{a}=\left[a_{1}, a_{2}, \ldots, a_{\ell}\right]$ is a row matrix and $\mathbf{b}=\left[b_{1}, b_{2}, \ldots, b_{\ell}\right]^{t}$ is a column.

\section{Fundamental techniques}

In this section we collect a small number of results for use later on. We will frequently use a consequence of [23, Coroll. 2.3] and Haagerup's theorem [3, 5.4.7]. Before stating it, we need some more notation.

When $X, Y$ are positive semidefinite $\ell \times \ell$ matrices (over $\mathbb{C}$ ) we use $\operatorname{tgm}(X, Y)$ for the trace of $(\sqrt{X} Y \sqrt{X})^{1 / 2}$, a quantity called the 'tracial geometric mean' in [23. For $u=\mathbf{a} \odot \mathbf{b} \in A \otimes A$ and $\phi \in \mathbb{S}(A)$ we let $\mathcal{Q}(\mathbf{b}, \phi)$ be the positive semidefinite $\ell \times \ell$ matrix defined by

$$
\mathcal{Q}(\mathbf{b}, \phi)=\left(\phi\left(b_{i}^{*} b_{j}\right)\right)_{i, j=1}^{\ell} .
$$

Similarly $\mathcal{Q}\left(\mathbf{a}^{*}, \phi\right)=\left(\phi\left(a_{i} a_{j}^{*}\right)\right)_{i, j=1}^{\ell}$.

Lemma 2.1. Let $A$ be a $C^{*}$-algebra and $u=\sum_{j=1}^{\ell} a_{j} \otimes b_{j} \in A \otimes A$. Then

$$
\|u\|_{h}=\sup \left\{\operatorname{tgm}\left(\mathcal{Q}\left(\mathbf{a}^{*}, \phi_{1}\right), \mathcal{Q}\left(\mathbf{b}, \phi_{2}\right)\right): \phi_{1}, \phi_{2} \in \operatorname{co}(\mathbb{P}(A))\right\},
$$

where $\operatorname{co}(\mathbb{P}(A))$ means the convex hull of $\mathbb{P}(A)$.

Proof. By separate homogeneity (of order $1 / 2$ ) of tgm in each variable, we can replace $\operatorname{co}(\mathbb{P}(A))$ by $\operatorname{co}(\mathbb{P}(A) \cup\{0\})$ without changing the supremum above. By the Krein-Milman theorem, we can further replace $\operatorname{co}(\mathbb{P}(A) \cup\{0\})$ by the quasi-state space $E(A)=\{t \phi: \phi \in \mathbb{S}(A), 0 \leq t \leq 1\}$ without affecting the supremum in (2.1). [We rely here, and elsewhere, on the joint weak*-continuity of the expression $\operatorname{tgm}\left(\mathcal{Q}\left(\mathbf{a}^{*}, \phi_{1}\right), \mathcal{Q}\left(\mathbf{b}, \phi_{2}\right)\right)$ for $\phi_{1}, \phi_{2} \in E(A)$ and fixed $\mathbf{a}$, b. This continuity is evident from the continuity of the square root of a positive semidefinite matrix.]

Consider a faithful embedding $A \subset \mathcal{B}(H)$ for some $H$. By injectivity we can compute the norm $\|u\|_{h}$ in $\mathcal{B}(H) \otimes_{h} \mathcal{B}(H)$, where it is the same as the norm of the elementary operator $\theta(u)$ on $\mathcal{K}(H)$ given by $x \mapsto \sum_{j=1}^{\ell} a_{j} x b_{j}$ (Haagerup's theorem). By [23, Coroll. 2.3], the completely bounded norm of the elementary operator is

$$
\|u\|_{h}=\|\theta(u)\|_{c b}=\sup \left\{\operatorname{tgm}\left(\mathcal{Q}\left(\mathbf{a}^{*}, \phi_{1}\right), \mathcal{Q}\left(\mathbf{b}, \phi_{2}\right)\right): \phi_{1}, \phi_{2} \in \mathbb{F}_{f}(\mathcal{K}(H))\right\},
$$

where it is to be understood that $\phi_{1}$ and $\phi_{2}$ are extended (uniquely) to states of the multiplier algebra $M(\mathcal{K}(H))=\mathcal{B}(H)$. Thus $\phi_{1}$ and $\phi_{2}$ are convex combinations of vector states of $\mathcal{B}(H)$. Since such states are weak*-dense in $\mathbb{S}(\mathcal{B}(H)$ ) (by [16, 3.4.1]), we can replace $\mathbb{F}_{f}(\mathcal{K}(H))$ by $\mathbb{S}(\mathcal{B}(H))$ in this formula. By the separate homogeneity, we can further replace $\mathbb{S}(\mathcal{B}(H))$ by $E(\mathcal{B}(H))$. Restricting $\phi_{1}, \phi_{2} \in E(\mathcal{B}(H))$ to $A$ we get quasi-states of $A$. So $\|u\|_{h}$ is at least the supremum in (2.1). As all elements of $E(A)$ are restrictions of elements of $E(\mathcal{B}(H))$, we have the opposite inequality also.

The following argument is similar to one in the proof of $(1) \Rightarrow(2)$ in $[5$, Th. 3.3]. In the course of the proof we will be considering the open map $\beta: \mathbb{P}(A) \rightarrow \hat{A}$ given by $\beta(\psi)=\pi_{\psi}$ (the equivalence class of the GNS representation associated to $\psi$ ). Recall that states $\phi \in \mathbb{F}_{f}(A)$ are convex combinations $\sum_{i=1}^{n} t_{i} \psi_{i}$ where $\psi_{i} \in \mathbb{P}(A)$ and $\beta\left(\psi_{i}\right)$ is constant $(1 \leq i \leq n)$. The assignment $\tilde{\beta}(\phi)=\beta\left(\psi_{1}\right)$ gives a well-defined map $\tilde{\beta}: \mathbb{F}_{f}(A) \rightarrow \hat{A}$ (see [11, p. 134]). 
Lemma 2.2. Let $A$ be a $C^{*}$-algebra and $Q$ a primal ideal of $A$. Given any two states $\phi_{1}, \phi_{2} \in \mathbb{S}(A / Q)$, there are commonly indexed nets $\left(\phi_{1, \alpha}\right)_{\alpha}$ and $\left(\phi_{2, \alpha}\right)_{\alpha}$ in the finite type I factorial states $\mathbb{F}_{f}(A)$ of $A$ with the properties

(i) $\lim _{\alpha} \phi_{i, \alpha}=\phi_{i}(i=1,2)$,

(ii) $\tilde{\beta}\left(\phi_{1, \alpha}\right)=\tilde{\beta}\left(\phi_{2, \alpha}\right)$ for all $\alpha$.

Moreover, if $\phi_{1}, \phi_{2} \in \mathbb{P}(A / Q)$, we can take all $\phi_{i, \alpha} \in \mathbb{P}(A)$.

Proof. We choose the index set for the nets to be a weak*-neighbourhood base $\mathcal{U}$ of open convex neighbourhoods of the origin in $A^{*}$ (ordered by reverse inclusion). For each $U \in \mathcal{U}$ choose finite convex combinations $\psi^{i, U}=\sum_{k=1}^{N_{U}} t_{k}^{i, U} \psi_{k}^{i, U} \in \phi_{i}+U / 2$, where $t_{k}^{i, U} \in[0,1], \sum_{k=1}^{N_{U}} t_{k}^{i, U}=1, \psi_{k}^{i, U} \in \mathbb{P}(A / Q)$ for $1 \leq k \leq N_{U}$ and $i=1,2$.

By primality of $Q$, the $2 N_{U}$ open sets $\beta\left(\mathbb{P}(A) \cap\left(\psi_{k}^{i, U}+U / 2\right)\right)$ must have nonempty intersection. Choose $\pi_{U} \in \hat{A}$ in this intersection. Then, for $1 \leq k \leq N_{U}$ and $i=1,2$, there exist $\tilde{\psi}_{k}^{i, U} \in \mathbb{P}(A)$ with $\tilde{\psi}_{k}^{i, U}-\psi_{k}^{i, U} \in U / 2$ and $\beta\left(\tilde{\psi}_{k}^{i, U}\right)=\pi_{U}$. Take $\phi_{i, \alpha}=\phi_{i, U}=\sum_{k=1}^{N_{U}} t_{k}^{i, U} \tilde{\psi}_{k}^{i, U} \in \phi_{i}+U$ to get the desired nets.

If we start with pure states $\phi_{1}, \phi_{2} \in \mathbb{P}(A / Q)$, there is no need for convex combinations and the argument becomes simpler.

Note that by [11, Proposition 2.1], condition (ii) above is equivalent to $\left(\phi_{1, \alpha}+\phi_{2, \alpha}\right) / 2 \in \mathbb{F}_{f}(A)$.

Lemma 2.3. Let $X$ and $Y$ be operator spaces and $\phi: X \rightarrow Y$ be a complete contraction. Then $\phi^{(n)} \otimes \phi^{(n)}: M_{n}(X) \otimes_{h} M_{n}(X) \rightarrow M_{n}(Y) \otimes_{h} M_{n}(Y)$ is contractive for each $n \geq 1$.

Proof. The norm of a tensor $u \in M_{n}(X) \otimes_{h} M_{n}(X)$ is the infimum of $\|\mathbf{a}\|\|\mathbf{b}\|$ over all representations $u=\sum_{j=1}^{\ell} a_{j} \otimes b_{j}$, where $\|\mathbf{a}\|$ means the norm of the row $\left[a_{1}, a_{2}, \ldots, a_{\ell}\right] \in M_{1, \ell}\left(M_{n}(X)\right) \subseteq M_{n \ell}(X)$ and $\|\mathbf{b}\|$ means the norm of the column $\left[b_{1}, b_{2}, \ldots, b_{\ell}\right]^{t}$. (See, for example, [19, p. 240].) Applying $\phi^{(n)}$ to each $a_{j}, b_{j}$ gives a representation of $\left(\phi^{(n)} \otimes \phi^{(n)}\right)(u) \in M_{n}(Y) \otimes M_{n}(Y)$. Since $\phi^{(n \ell)}$ is a complete contraction, $\left\|\left(\phi^{(n)} \otimes \phi^{(n)}\right)(u)\right\|_{h} \leq\|\mathbf{a}\|\|\mathbf{b}\|$.

Here is a generalisation of [10, Lemma 5], with a more succinct proof.

Lemma 2.4. Let $b_{j}(1 \leq j \leq N)$ be orthogonal, positive elements of norm one in a $C^{*}$-algebra $A$ (that is, $b_{j} \geq 0,\left\|b_{j}\right\|=1$ and $b_{j} b_{k}=0$ for $j \neq k, 1 \leq j, k \leq N$ ) and let $X$ denote their linear span. Let $d_{j}(1 \leq j \leq N)$ be orthogonal positive elements of a $C^{*}$-algebra $B$ and let $Y$ denote their linear span. Assume $\left\|d_{j}\right\| \leq 1$ for $1 \leq j \leq N$.

We can define a linear map $\phi: X \rightarrow Y$ by $\phi\left(b_{j}\right)=d_{j}$, and it has the following properties:

(i) $\|\phi\| \leq 1$.

(ii) The map $\phi^{(n)} \otimes \phi^{(n)}: M_{n}(X) \otimes_{h} M_{n}(X) \rightarrow M_{n}(Y) \otimes_{h} M_{n}(Y)$ (with Haagerup tensor norms in each case) has norm at most one.

(iii) If $\left\|d_{j}\right\|=1$ for each $j$, then $\phi^{(n)} \otimes \phi^{(n)}$ is an isometry between $M_{n}(X) \otimes_{h}$ $M_{n}(X)$ and $M_{n}(Y) \otimes_{h} M_{n}(Y)$.

Proof. The fact that $\phi$ is contractive is proved in [10, Lemma 5]. As $\phi$ has range in the commutative $C^{*}$-algebra generated by $d_{1}, d_{2}, \ldots, d_{N}$, it must be completely contractive ([19, p. 107]). By Lemma 2.3 we deduce $\left\|\phi^{(n)} \otimes \phi^{(n)}\right\| \leq 1$. 
To get the last statement, we can apply the same arguments to the inverse map from the span of $d_{1}, d_{2}, \ldots, d_{N}$ to the commutative $C^{*}$-algebra generated by $b_{1}, b_{2}, \ldots, b_{N}$.

\section{The nOn-Unital CEntral HaAgerup tensor Product}

In this section we extend the theory of the central Haagerup tensor product to cover the case when the algebra need not be unital. This is desirable for two reasons: first because there are interesting non-unital examples such as $C^{*}$-algebras from non-discrete groups, and second because we shall soon wish to consider the stabilization $A \otimes \mathcal{K}(H)$ of a $C^{*}$-algebra $A$. Let $A$ be a (possibly non-unital) $C^{*}$ algebra.

Definition 3.1. Let $J_{A}$ be the closure in $A \otimes_{h} A$ of the linear span of the elements of the form $a z \otimes b-a \otimes z b$ with $a, b \in A, z \in Z(M(A))$, the centre of the multiplier algebra $M(A)$. (In fact $J_{A}$ is an ideal.)

We define $A \otimes_{Z, h} A$ to be the quotient $\left(A \otimes_{h} A\right) / J_{A}$, and we denote the quotient norm by $\|\cdot\|_{Z, h}$.

Note that this agrees with the usual definition of $A \otimes_{Z, h} A$ when $A$ is unital (in which case $M(A)=A)$.

Notation 3.2. We recall that the map $\theta: A \otimes A \rightarrow \mathrm{CB}(A)$ given by

$$
\theta\left(\sum_{j=1}^{\ell} a_{j} \otimes b_{j}\right)(x)=\sum_{j=1}^{\ell} a_{j} x b_{j}
$$

is contractive in the Haagerup tensor norm $\left(\|\theta(u)\|_{c b} \leq\|u\|_{h}\right)$. Hence it extends to a contraction on the completed tensor product $\theta: A \otimes_{h} A \rightarrow \mathrm{CB}(A)$, and it is evident that $\theta\left(J_{A}\right)=0$. Thus the map $\theta$ induces a contraction $\theta_{Z}: A \otimes_{Z, h} A \rightarrow \mathrm{CB}(A)$, where $\theta_{Z}\left(u+J_{A}\right)=\theta(u)$ for $u \in A \otimes_{h} A$, and $\left\|\theta_{Z}\left(u+J_{A}\right)\right\|_{c b} \leq\left\|u+J_{A}\right\|_{Z, h}$. For convenience we will frequently write $\theta_{Z}(u)$ and $\|u\|_{Z, h}$ where it is to be understood that $u$ should be replaced by the coset $u+J_{A}$.

Lemma 3.3. For each $G \in \operatorname{Glimm}(A), A \otimes_{h} G+G \otimes_{h} A \supseteq J_{A}$.

Proof. Let $G \in \operatorname{Glimm}(A)$. In the proof of [10, Lemma 10], elaborating an argument from [3, p. 88], it is shown that there is a maximal ideal $J$ of $Z(M(A))$ such that $J A \subseteq G$. Let $\phi_{J}: Z(M(A)) \rightarrow \mathbb{C}$ be the multiplicative linear functional with kernel $J$, and let $z \in Z(M(A))$. Then $z-\phi_{J}(z) 1 \in J$, and so for $a, b \in A$ we have

$$
\begin{aligned}
a z \otimes b-a \otimes z b & =\left(a z-\phi_{J}(z) a\right) \otimes b-a \otimes\left(z b-\phi_{J}(z) b\right) \\
& =\left(\left(z-\phi_{J}(z) 1\right) a\right) \otimes b-a \otimes\left(\left(z-\phi_{J}(z) 1\right) b\right) \\
& \in A \otimes_{h} G+G \otimes_{h} A .
\end{aligned}
$$

As $A \otimes_{h} G+G \otimes_{h} A$ is a closed ideal in $A \otimes_{h} A$ (by [1, Corollary 2.6]), the result follows.

The following lemma is presumably well known, but we have been unable to locate a reference.

Lemma 3.4. Let $T$ be a completely regular (Hausdorff) space, $Y \subseteq X$ a compact subset and $N_{1}, N_{2}, \ldots, N_{m}$ a finite open cover of $Y$. Then there exist $\mathbb{R}$-valued continuous functions $f_{1}, \ldots, f_{m}, f_{m+1}$ on $T$ with $0 \leq f_{j} \leq 1(1 \leq j \leq m+1), f_{j}$ vanishes outside $N_{j}(1 \leq j \leq m), \sum_{j=1}^{m} f_{j}=1$ on $Y$ and $f_{m+1}=1-\sum_{j=1}^{m} f_{j}$. 
Proof. As $T$ is a completely regular (Hausdorff) space there is a compact Hausdorff space $T^{\prime}$ that contains it. There are open $N_{j}^{\prime} \subseteq T^{\prime}$ so that $N_{j}^{\prime} \cap T=N_{j}$. As $\left\{N_{j}^{\prime}: 1 \leq j \leq m\right\} \cup\left\{T^{\prime} \backslash Y\right\}$ is an open cover of $T^{\prime}$, there are continuous functions $f_{j}^{\prime}: T^{\prime} \rightarrow \mathbb{R}(1 \leq j \leq m+1)$ with $\sum_{j=1}^{m+1} f_{j}^{\prime}=1,0 \leq f_{j}^{\prime} \leq 1(1 \leq j \leq m+1)$, $f_{j}^{\prime}$ vanishes outside $N_{j}^{\prime}$ and $f_{m+1}^{\prime}$ vanishes on $Y$. Let $f_{j}$ be the restriction of $f_{j}^{\prime}$ to $T$.

The next result is a direct generalisation of [22, Theorem 1] to include the nonunital case. Recall that from [1, Corollary 2.6], for $I$ an ideal in $A$, the quotient $\left(A \otimes_{h} A\right) /\left(A \otimes_{h} I+I \otimes_{h} A\right)$ is isometrically isomorphic to $(A / I) \otimes_{h}(A / I)$. For $u \in A \otimes_{h} A$ we let $u^{I}$ denote the image of $u$ in the quotient.

Theorem 3.5. Let $A$ be a $C^{*}$-algebra and let $u \in A \otimes_{h} A$. Then

$$
\|u\|_{Z, h}=\sup \left\{\left\|u^{G}\right\|_{h}: G \in \operatorname{Glimm}(A)\right\} .
$$

Hence $J_{A}=\bigcap\left\{A \otimes_{h} G+G \otimes_{h} A: G \in \operatorname{Glimm}(A)\right\}$.

Proof. It is enough to prove equality when $u$ has the form $u=\sum_{i=1}^{\ell} a_{i} \otimes b_{i}\left(a_{i}, b_{i} \in\right.$ $A)$. Let $\alpha=\sup \left\{\left\|u^{G}\right\|_{h}: G \in \operatorname{Glimm}(A)\right\}$. From Lemma 3.3 it is clear that $\|u\|_{Z, h} \geq \alpha$. Suppose $\varepsilon>0$ is given. Set

$$
\begin{aligned}
X= & \left\{P \in \operatorname{Prim}(A):\left\|\sum_{i=1}^{\ell}\left(a_{i} a_{i}^{*}+P\right)\right\| \geq \varepsilon / 2\right\} \\
& \cup\left\{P \in \operatorname{Prim}(A):\left\|\sum_{i=1}^{\ell}\left(b_{i}^{*} b_{i}+P\right)\right\| \geq \varepsilon / 2\right\} .
\end{aligned}
$$

Then $X$ is compact, being a union of two compact sets (by [16, 3.3.7]). Let $Y=\phi_{A}(X)$, where $\phi_{A}: \operatorname{Prim}(A) \rightarrow \operatorname{Glimm}(A)$ is the complete regularisation map. Then $Y$ is a compact subset of $\operatorname{Glimm}(A)$.

For each $G \in Y$ there exists by [13, Lemma 2.3] an invertible $\ell \times \ell$ matrix $S$ such that if $\left(a_{i}^{\prime}\right)=\left(a_{i}\right) S^{-1}$ and $\left(b_{i}^{\prime}\right)=S\left(b_{i}\right)$, then

$$
\left\|\sum_{i=1}^{\ell}\left(a_{i}^{\prime} a_{i}^{\prime *}+G\right)\right\|,\left\|\sum_{i=1}^{\ell}\left(b_{i}^{\prime *} b_{i}^{\prime}+G\right)\right\|<\alpha+\frac{\varepsilon}{2} .
$$

For $x \in A$, the norm function $G \mapsto\|x+G\|$ is upper semicontinuous on $\operatorname{Glimm}(A)$ (see the first paragraph of the proof of [24, Theorem 3.1] or [14, Corollary 1.9] for the general, non-unital, case). By this upper semicontinuity there is a neighbourhood $N$ of $G$ such that

$$
\left\|\sum_{i=1}^{\ell}\left(a_{i}^{\prime} a_{i}^{\prime *}+G^{\prime}\right)\right\|,\left\|\sum_{i=1}^{\ell}\left(b_{i}^{\prime *} b_{i}^{\prime}+G^{\prime}\right)\right\|<\alpha+\frac{\varepsilon}{2}
$$

for all $G^{\prime} \in N$. Thus by compactness of $Y$ there exist open subsets $\left\{N_{j}\right\}_{j=1}^{m}$ of $\operatorname{Glimm}(A)$ and invertible $\ell \times \ell$ matrices $\left\{S_{j}\right\}_{j=1}^{m}$ such that the $N_{j}$ cover $Y$, and if $G \in N_{j}$, then

$$
\left\|\sum_{i=1}^{\ell}\left(a_{i}^{j} a_{i}^{j *}+G\right)\right\|,\left\|\sum_{i=1}^{\ell}\left(b_{i}^{j *} b_{i}^{j}+G\right)\right\|<\alpha+\frac{\varepsilon}{2},
$$

where $\left(a_{i}^{j}\right)=\left(a_{i}\right) S_{j}^{-1}$ and $\left(b_{i}^{j}\right)=S_{j}\left(b_{i}\right)$. 
As $\operatorname{Glimm}(A)$ is a completely regular (Hausdorff) space, we can apply Lemma 3.4 to find continuous $f_{j}: \operatorname{Glimm}(A) \rightarrow \mathbb{R}(1 \leq j \leq m+1)$ with $0 \leq f_{j} \leq 1$ $(1 \leq j \leq m+1), f_{j}$ vanishes outside $N_{j}(1 \leq j \leq m), \sum_{j=1}^{m} f_{j}=1$ on $Y$ and $f_{m+1}=1-\sum_{j=1}^{m} f_{j}$. Let $z_{1}, \ldots, z_{m+1}$ be the elements of $Z(M(A))$ corresponding to $f_{1}, \ldots, f_{m+1}$ under the Dauns-Hofmann Theorem ([15, or see for instance [20, $\S$ A.3]). Then for $x \in A$ and $P \in \operatorname{Prim}(A)$ we have $z_{j} a+P=f_{j}\left(\phi_{A}(P)\right) a+P$ $(1 \leq j \leq m+1)$. For $x \in A$ and $G \in \operatorname{Glimm}(A)$ we have $z_{j} a+G=f_{j}(G) a+G$ $(1 \leq j \leq m+1)$ since $z_{j} a-f_{j}(G) a$ belongs to all $P \in \operatorname{Prim}(A)$ with $P \supseteq G$.

Set

$$
v=\sum_{j=1}^{m} \sum_{i=1}^{\ell} a_{i}^{j} z_{j}^{1 / 2} \otimes z_{j}^{1 / 2} b_{i}^{j} \text { and } w=\sum_{i=1}^{\ell} a_{i} z_{m+1}^{1 / 2} \otimes z_{m+1}^{1 / 2} b_{i} .
$$

Then for $G \in \operatorname{Glimm}(A)$

$$
\begin{aligned}
\left\|\sum_{j=1}^{m} \sum_{i=1}^{\ell}\left(z_{j} a_{i}^{j} a_{i}^{j *}+G\right)\right\| & =\left\|\sum_{j=1}^{m} f_{j}(G) \sum_{i=1}^{\ell}\left(a_{i}^{j} a_{i}^{j *}+G\right)\right\| \\
& <\alpha+\frac{\varepsilon}{2}
\end{aligned}
$$

and similarly for $G^{\prime} \in \operatorname{Glimm}(A)$,

$$
\left\|\sum_{j=1}^{m} \sum_{i=1}^{\ell}\left(z_{j} b_{i}^{j *} b_{i}^{j}+G^{\prime}\right)\right\|<\alpha+\frac{\varepsilon}{2}
$$

Hence

$$
\|v\|_{h} \leq\left\|\sum_{j=1}^{m} \sum_{i=1}^{\ell} z_{j} a_{i}^{j} a_{i}^{j *}\right\|^{1 / 2}\left\|\sum_{j=1}^{m} \sum_{i=1}^{\ell} z_{j} b_{i}^{j *} b_{i}^{j}\right\|^{1 / 2}<\alpha+\frac{\varepsilon}{2}
$$

(since $\bigcap\{G: G \in \operatorname{Glimm}(A)\}=\{0\}$ ).

Furthermore, for $P \in \operatorname{Prim}(A)$

$$
\left\|\sum_{i=1}^{\ell}\left(z_{m+1} a_{i} a_{i}^{*}+P\right)\right\|=f_{m+1}\left(\phi_{A}(P)\right)\left\|\sum_{i=1}^{\ell}\left(a_{i} a_{i}^{*}+P\right)\right\|<\frac{\varepsilon}{2},
$$

since $f_{m+1}$ is supported in $\operatorname{Glimm}(A) \backslash Y$.

Similarly for $P \in \operatorname{Prim}(A)$,

$$
\left\|\sum_{i=1}^{\ell}\left(z_{m+1} b_{i}^{*} b_{i}+P\right)\right\|<\frac{\varepsilon}{2}
$$

Hence

$$
\|w\|_{h} \leq\left\|\sum_{i=1}^{\ell} z_{m+1} a_{i} a_{i}^{*}\right\|^{1 / 2}\left\|\sum_{i=1}^{\ell} z_{m+1} b_{i}^{*} b_{i}\right\|^{1 / 2}<\frac{\varepsilon}{2} .
$$

Finally we show that $u-(v+w) \in J_{A}$. For this it is convenient to work inside $M(A) \otimes_{h} M(A)$ using the natural isometric isomorphism $A \otimes_{h} A \hookrightarrow M(A) \otimes_{h} M(A)$. 
Then

$$
\begin{aligned}
u-(v+w) & =u-\sum_{j=1}^{m} u\left(z_{j}^{1 / 2} \otimes z_{j}^{1 / 2}\right)-u\left(z_{m+1}^{1 / 2} \otimes z_{m+1}^{1 / 2}\right) \\
& =u\left(1-\sum_{j=1}^{m+1} z_{j}^{1 / 2} \otimes z_{j}^{1 / 2}\right) \\
& =u\left(\sum_{j=1}^{m+1}\left(z_{j}^{1 / 2} \otimes 1\right)\left(z_{j}^{1 / 2} \otimes 1-1 \otimes z_{j}^{1 / 2}\right)\right) .
\end{aligned}
$$

But $u\left(z_{j}^{1 / 2} \otimes 1-1 \otimes z_{j}^{1 / 2}\right) \in J_{A}$ for all $j$, so $u-(v+w) \in J_{A}$.

Hence $\|u\|_{Z, h} \leq\|v+w\|_{h}<\alpha+\varepsilon$, as required.

A fact that we will often use is stated here for easy reference and can be found in $[3,5.3 .12,5.4 .10]$.

Proposition 3.6. For $A$ a $C^{*}$-algebra and $u \in A \otimes A,\|\theta(u)\|_{c b}=\sup \left\{\left\|u^{P}\right\|_{h}\right.$ : $P \in \operatorname{Prim}(A)\}$.

The next result is proved in [22, Proposition 3], but we give a rather different proof by using Lemma 2.1

Proposition 3.7. Let $A$ be a $C^{*}$-algebra and let $u \in A \otimes_{h} A$. Then

$$
\begin{aligned}
\|\theta(u)\|_{c b} & =\sup \left\{\left\|u^{Q}\right\|_{h}: Q \in \operatorname{Primal}(A)\right\} \\
& =\sup \left\{\left\|u^{Q}\right\|_{h}: Q \in \operatorname{Min}-\operatorname{Primal}(A)\right\} .
\end{aligned}
$$

Proof. Since $\theta_{Z}$ is contractive, and so are the canonical maps $A \otimes_{h} A \rightarrow(A / Q) \otimes_{h}$ $(A / Q)$, it is sufficient to prove the result when $u=\sum_{j=1}^{\ell} a_{j} \otimes b_{j} \in A \otimes A$.

Since $\operatorname{Prim}(A) \subseteq \operatorname{Primal}(A)$, we get an inequality from Proposition 3.6.

By Lemma 2.1 if $Q \in \operatorname{Primal}(A)$ and $\varepsilon>0$, then there exist $\phi_{1}, \phi_{2} \in \operatorname{co}(\mathbb{P}(A / Q))$ with

$$
\operatorname{tgm}\left(\mathcal{Q}\left(\mathbf{a}^{*}, \phi_{1}\right), \mathcal{Q}\left(\mathbf{b}, \phi_{2}\right)\right) \geq\left\|u^{Q}\right\|_{h}-\varepsilon .
$$

By Lemma 2.2 there are nets $\left(\phi_{1, \alpha}\right)_{\alpha}$ and $\left(\phi_{2, \alpha}\right)_{\alpha}$ in $\mathbb{F}_{f}(A)$ satisfying $\tilde{\beta}\left(\phi_{1, \alpha}\right)=$ $\tilde{\beta}\left(\phi_{2, \alpha}\right)($ all $\alpha)$ and $\lim _{\alpha} \phi_{i, \alpha}=\phi_{i}(i=1,2)$. For each $\alpha$, let $P_{\alpha}=\operatorname{ker} \tilde{\beta}\left(\phi_{1, \alpha}\right)$ and then $\phi_{1, \alpha}, \phi_{2, \alpha} \in \operatorname{co} \mathbb{P}\left(A / P_{\alpha}\right)$. So, by Lemma 2.1] and Proposition 3.6.

$$
\operatorname{tgm}\left(\mathcal{Q}\left(\mathbf{a}^{*}, \phi_{1, \alpha}\right), \mathcal{Q}\left(\mathbf{b}, \phi_{2, \alpha}\right)\right) \leq\left\|u^{P_{\alpha}}\right\|_{h} \leq\|\theta(u)\|_{c b} .
$$

Taking limits we get $\left\|u^{Q}\right\|_{h}-\varepsilon \leq\|\theta(u)\|_{c b}$. This establishes the first equality.

The second follows because each primal ideal contains a minimal primal and $\left\|u^{Q}\right\|_{h}$ increases as $Q$ decreases.

The following generalises [10, Theorem 8] and [22, Theorem 4, Corollary 6 (ii)].

Theorem 3.8. Let $A$ be a $C^{*}$-algebra and $\theta_{Z}: A \otimes_{Z, h} A \rightarrow C B(A)$ the linear contraction given by $\theta_{Z}\left(a \otimes b+J_{A}\right)(x)=a x b$. Then

(i) $\theta_{Z}$ is injective if and only if each $G \in \operatorname{Glimm}(A)$ is a 2-primal ideal of $A$.

(ii) $\theta_{Z}$ is an isometry if and only if each $G \in \operatorname{Glimm}(A)$ is a primal ideal of $A$.

Proof. (i) This can be shown as in [22, Corollary 6] using Theorem 3.5 in place of [22, Theorem 1]. 
(ii) If each Glimm ideal of $A$ is primal, then we can see that $\theta_{Z}$ is isometric by Theorem 3.5 and Proposition 3.7. (See [22, Theorem 4] for the unital case.)

The converse follows as in [10, Theorem 17], using Theorem 3.5] in place of [22, Theorem 1].

The family of $C^{*}$-algebras for which every Glimm ideal is primal includes the following: von Neumann algebras [5, Proposition 4.3], quotients of $A W^{*}$-algebras [21, Lemma 2.8], prime $C^{*}$-algebras, and the $C^{*}$-algebras of amenable discrete groups [18. Let $G$ be a simply connected nilpotent Lie group, $\mathfrak{g}$ the Lie algebra of $G$ and $\mathfrak{z}$ the centre of $\mathfrak{g}$. Then every Glimm ideal of $C^{*}(G)$ is primal if the maximal coadjoint orbit dimension in $\mathfrak{g}^{*}$ equals $\operatorname{dim}(\mathfrak{g} / \mathfrak{z})$. Moreover, the converse holds in the case where $G$ is 2 -step nilpotent [ 6 . Thus, for example, every Glimm ideal is primal in the case where $G$ is the continuous Heisenberg group and also in the case $G=W_{n}$ ( $n$ even) where $W_{n}$ is the "universal" simply connected, two-step nilpotent Lie group studied in [8, Section 2]. Up to topological isomorphism, there are 24 simply connected, nilpotent Lie groups of dimension 6 (excluding those which are direct products of lower dimensional groups). Of these, 21 have a group $C^{*}$-algebra for which every Glimm ideal is primal [8, p. 292].

We note briefly that the main result of [10, Section 4] also extends to the nonunital case.

Theorem 3.9. Let $A$ be a $C^{*}$-algebra. Fix $\ell \geq 1$. Then

$$
\left\|\theta_{Z}(u)\right\|_{c b}=\|u\|_{Z, h}
$$

holds for each $u=\sum_{j=1}^{\ell} a_{j} \otimes b_{j} \in A \otimes A$ if and only if every Glimm ideal in $A$ is $\left(\ell^{2}+1\right)$-primal.

Proof. The proof of sufficiency of the condition that every Glimm ideal in $A$ is $\left(\ell^{2}+1\right)$-primal is as in [10, Proposition 14], using Theorem [3.5 in place of [22, Theorem 1].

The proof of the converse is as in [10, Theorem 17]. In the course of proving Theorem 5.1 below, we will generalise the main ideas of the proof of 10 , Theorem $17]$.

Example 3.10. In [10, Example 12] an example is given of a $C^{*}$-algebra $A$ where each Glimm ideal of $A$ is primal but there is a Glimm ideal in $M(A)$ that is not 2-primal. In this case the map $\theta_{Z}: A \otimes_{Z, h} A \rightarrow \mathrm{CB}(A)$ is isometric (by Theorem 3.8 (ii)) but the linear contraction $\Theta_{Z}: M(A) \otimes_{Z, h} M(A) \rightarrow \mathrm{CB}(A)$ given by

$$
\Theta_{Z}\left(c \otimes d+J_{M(A)}\right)(x)=c x d
$$

is not even injective (by Theorem 3.8 (i)).

\section{The constants $L(A)$ and $L^{\prime}(A)$ And basic properties}

In this section we define the constants $L(A)$ and $L^{\prime}(A)$ associated to a $C^{*}$ algebra $A$ and we construct combinatorially defined models called simplicial-spoke $C^{*}$-algebras which will be useful for determining $L^{\prime}(A)$ for general $\mathrm{C}^{*}$-algebras in later sections. We also give some basic results for later use. 
Definition 4.1. For a (unital or non-unital) $C^{*}$-algebra $A$, define

$$
L(A)=\inf \left\{C>0:\|u\|_{Z, h} \leq C\left\|\theta_{Z}(u)\right\|_{c b} \forall u \in A \otimes A\right\},
$$

where $\theta_{Z}: A \otimes_{Z, h} A \rightarrow C B(A)$ is the canonical map introduced in Notation 3.2

We define

$$
L^{\prime}(A)=\sup _{n \geq 1} L\left(M_{n}(A)\right) .
$$

Remark 4.2. By a density argument, we could equally define $L(A)=\inf \{C>0$ : $\left.\|u\|_{Z, h} \leq C\left\|\theta_{Z}(u)\right\|_{c b} \forall u \in A \otimes_{Z, h} A\right\}$ (using the completion). It follows that if $A$ has a Glimm ideal that is not 2-primal (so that $\theta_{Z}$ is not injective by Theorem 3.8), then $L(A)=\infty$. If $L(A)<\infty$, then $\theta_{Z}$ is an isomorphism onto its range, and by completeness of $A \otimes_{Z, h} A$, the range of $\theta_{Z}$ is closed. Conversely if $\theta_{Z}$ is injective and has closed range, then $L(A)<\infty$ (by the open mapping theorem).

If all Glimm ideals of $A$ are primal, then we know $L(A)=L^{\prime}(A)=1$ by Theorem 3.8 (ii) (and remarks in the Introduction concerning $\operatorname{Glimm}\left(M_{n}(A)\right)$ and $\left.\operatorname{Primal}\left(M_{n}(A)\right)\right)$.

Proposition 4.3. Let $A$ be a $C^{*}$-algebra. Then $L\left(M_{n}(A)\right) \leq L\left(M_{n+1}(A)\right)$ for $n \geq 1$.

Proof. There is a $*$-algebra embedding $j_{n}: M_{n}(A) \rightarrow M_{n+1}(A)$ adding a row and column of zeros to $x \in M_{n}(A)$ to get $j_{n}(x) \in M_{n+1}(A)$. Moreover for $u \in M_{n}(A) \otimes$ $M_{n}(A), I$ any ideal of $A$, and $q_{I}: A \rightarrow A / I$ the quotient map, $\left(q_{I}\right)^{(n)}: M_{n}(A)=$ $A \otimes M_{n} \rightarrow A / I \otimes M_{n}=M_{n}(A / I)=M_{n}(A) / M_{n}(I)$ is also a quotient and $j_{n} \circ$ $\left(q_{I}\right)^{(n)}=\left(q_{I}\right)^{(n+1)} \circ j_{n}$.

It follows that for $u \in M_{n}(A) \otimes M_{n}(A)$, if we let $v=\left(j_{n} \otimes j_{n}\right)(u) \in M_{n+1}(A) \otimes$ $M_{n+1}(A)$, then for each ideal $I$ of $A$ we have $\left\|u^{M_{n}(I)}\right\|_{h}=\left\|v^{M_{n+1}(I)}\right\|_{h}$. Hence

$$
\begin{aligned}
\|u\|_{Z, h} & =\sup \left\{\left\|u^{M_{n}(G)}\right\|_{h}: G \in \operatorname{Glimm}(A)\right\} \\
& =\sup \left\{\left\|v^{M_{n+1}(G)}\right\|_{h}: G \in \operatorname{Glimm}(A)\right\} \\
& =\|v\|_{Z, h}, \\
\left\|\theta_{Z}(u)\right\|_{c b} & =\sup \left\{\left\|u^{M_{n}(P)}\right\|_{h}: P \in \operatorname{Prim}(A)\right\} \\
& =\sup \left\{\left\|v^{M_{n+1}(P)}\right\|_{h}: P \in \operatorname{Prim}(A)\right\} \\
& =\left\|\theta_{Z}(v)\right\|_{c b},
\end{aligned}
$$

and it follows that $L\left(M_{n}(A)\right) \leq L\left(M_{n+1}(A)\right)$.

Proposition 4.4. Let $A$ and $B$ be $C^{*}$-algebras. Then

$$
L(A \oplus B)=\max (L(A), L(B))
$$

(interpreted in $[1, \infty])$. Moreover $L^{\prime}(A \oplus B)=\max \left(L^{\prime}(A), L^{\prime}(B)\right.$ ).

Proof. Let $q_{A}: A \oplus B \rightarrow A$ and $q_{B}: B \rightarrow A \oplus B$ be the coordinate projections. The irreducible representations of $A \oplus B$ are those of the form $\pi \circ q_{A}$ or $\sigma \circ q_{B}$ with $\pi$ an irreducible representation of $A$ and $\sigma$ an irreducible representation of $B$. Hence the primitive ideals of $A \oplus B$ are those of the form $P \oplus B$ and $A \oplus Q$ where $P \in \operatorname{Prim}(A), Q \in \operatorname{Prim}(B)$. As $A$ and $B$ are ideals of $A \oplus B, \operatorname{Prim}(A \oplus B)$ is topologically the disjoint union of $\operatorname{Prim}(A)$ and $\operatorname{Prim}(B)$.

It follows that the complete regularisation $\operatorname{Glimm}(A \oplus B)$ is the disjoint union $\operatorname{Glimm}(A) \cup \operatorname{Glimm}(B)$. In terms of ideals, $\operatorname{Glimm}(A \oplus B)=\{G \oplus B: G \in$ $\operatorname{Glimm}(A)\} \cup\{A \oplus H: H \in \operatorname{Glimm}(B)\}$. 
Let $u=\sum_{i=1}^{\ell}\left(a_{i}, b_{i}\right) \otimes\left(c_{i}, d_{i}\right) \in(A \oplus B) \otimes(A \oplus B)$, where $a_{i}, c_{i} \in A$ and $b_{i}, d_{i} \in B$ $(1 \leq i \leq n)$. Let $u_{A}=\sum_{i=1}^{\ell} a_{i} \otimes c_{i}\left(=\left(q_{A} \otimes q_{A}\right)(u)\right)$ and $u_{B}=\sum_{i=1}^{\ell} b_{i} \otimes d_{i}$. We first establish

$$
\|u\|_{Z, h}=\max \left(\left\|u_{A}\right\|_{Z, h},\left\|u_{B}\right\|_{Z, h}\right)
$$

and

$$
\|\theta(u)\|_{c b}=\max \left(\left\|\theta_{A}\left(u_{A}\right)\right\|_{c b},\left\|\theta_{B}\left(u_{B}\right)\right\|_{c b}\right)
$$

(where $\theta_{A}: A \otimes A \rightarrow \mathrm{CB}(A)$ is the usual map taking values in elementary operators on $A$, and $\theta_{B}, \theta=\theta_{A \oplus B}$ are similarly defined).

Suppose $G \in \operatorname{Glimm}(A)$. Then $A / G$ is canonically isomorphic to $(A \oplus B) /$ $(G \oplus B)$, and we deduce $\left\|u^{(G \oplus B)}\right\|_{h}=\left\|u_{A}^{(G)}\right\|_{h}$. Similarly we can verify $\left\|u^{(A \otimes H)}\right\|_{h}=$ $\left\|\left(u_{B}\right)^{(H)}\right\|_{h}$. Then (4.1) follows from Theorem 3.5.

On the other hand, replacing $G$ and $H$ in the above $\operatorname{arguments}$ by $P \in \operatorname{Prim}(A)$ and $Q \in \operatorname{Prim}(B)$ respectively, we obtain $\left\|u^{(P \oplus B)}\right\|_{h}=\left\|\left(u_{A}\right)^{(P)}\right\|_{h}$ and $\left\|u^{(A \oplus Q)}\right\|_{h}$ $=\left\|\left(u_{B}\right)^{(Q)}\right\|_{h}$. From this, (4.2) follows from Proposition 3.6.

Now let $0<L<L(A)$. Then there exists $w \in A \otimes A$ with

$$
\left\|\theta_{A}(w)\right\|_{c b}<\frac{1}{L}\|w\|_{Z, h} .
$$

Clearly there is $u \in(A \oplus B) \otimes(A \oplus B)$ such that $u_{A}=u$ and $u_{B}=0$. Then using (4.1) and (4.2), we have $\|\theta(u)\|_{c b}<\frac{1}{L}\|u\|_{Z, h}$. Thus $L(A \oplus B) \geq L(A)$, and similarly $L(A \oplus B) \geq L(B)$.

Now suppose $L(A)$ and $L(B)$ are both finite. Let $L=\max (L(A), L(B))$ and let $u \in(A \oplus B) \otimes(A \oplus B)$. Then

$$
\left\|\theta_{A}\left(u_{A}\right)\right\|_{c b} \geq \frac{1}{L}\left\|u_{A}\right\|_{Z, h} \text { and }\left\|\theta_{B}\left(u_{B}\right)\right\|_{c b} \geq \frac{1}{L}\left\|u_{B}\right\|_{Z, h} .
$$

Using (4.2), and then (4.1), we have

$$
\|\theta(u)\|_{c b} \geq \frac{1}{L} \max \left(\left\|u_{A}\right\|_{Z, h},\left\|u_{B}\right\|_{Z, h}\right)=\frac{1}{L}\|u\|_{Z, h} .
$$

Hence $L(A \oplus B) \leq L$, as required.

Since $M_{n}(A \oplus B) \cong M_{n}(A) \oplus M_{n}(B)$, the assertion about $L^{\prime}(A \oplus B)$ follows easily.

Corollary 4.5. Let $\left(A_{n}\right)_{n \geq 1}$ be a sequence of $C^{*}$-algebras. Let $A_{0}$ be the $c_{0}$ direct sum of the $A_{n}$. Then

$$
L\left(A_{0}\right)=\sup _{n \geq 1} L\left(A_{n}\right) \quad(\text { in }[1, \infty])
$$

and $L^{\prime}\left(A_{0}\right)=\sup _{n \geq 1} L^{\prime}\left(A_{n}\right)$.

Proof. For each $n, k \geq 1, M_{k}\left(A_{n}\right)$ is isomorphic to a direct summand of $M_{k}\left(A_{0}\right)$ and so $L\left(M_{k}\left(A_{n}\right)\right) \leq L\left(M_{k}\left(A_{0}\right)\right)$ by Proposition 4.4. Hence $L\left(A_{0}\right) \geq \sup _{n} L\left(A_{n}\right)$ (from $k=1$ ) and $L^{\prime}\left(A_{0}\right) \geq \sup _{n} L^{\prime}\left(A_{n}\right)$. Note that if either of the constants $L\left(A_{0}\right)$ or $L^{\prime}\left(A_{0}\right)$ is 1 , then we get the reverse inequalities by definition.

Let us agree that if $I_{n}$ is an ideal of $A_{n}$, then $I_{n}^{\prime}$ is the ideal of $A_{0}$ defined via the restriction on $x$ that $x_{n} \in I_{n}$.

Now suppose $k \geq 1$ and that $1 \leq L_{k}:=\sup _{n} L\left(M_{k}\left(A_{n}\right)\right)<\infty$. It suffices to show that $L\left(M_{k}\left(A_{0}\right)\right) \leq L_{k}$. 
We know that irreducible representations of $A_{0}$ must be of the form $\pi(x)=$ $\pi\left(\left(x_{i}\right)_{i=1}^{\infty}\right)=\pi_{n}\left(x_{n}\right)$ for some irreducible representation $\pi_{n}$ of $A_{n}$. Thus the primitive ideals of $A_{0}$ are those of the form $P_{n}^{\prime}$ for $P_{n} \in \operatorname{Prim}\left(A_{n}\right)$. Since each $A_{n}$ is a direct summand of $A_{0}$, it is easily seen that the Glimm ideals of $A_{0}$ are those of the form $G_{n}^{\prime}$ for $G_{n} \in \operatorname{Glimm}\left(A_{n}\right)$.

Now let $u=\sum_{i=1}^{\ell} a_{i} \otimes b_{i} \in M_{k}\left(A_{0}\right) \otimes M_{k}\left(A_{0}\right)$, and for each $n \geq 1$ let $u_{n}=$ $\sum_{i=1}^{\ell}\left(a_{i}\right)_{n} \otimes\left(b_{i}\right)_{n} \in M_{k}\left(A_{n}\right) \otimes M_{k}\left(A_{n}\right)$.

Observe that the canonical $*$-isomorphism

$$
x+M_{k}\left(I_{n}^{\prime}\right) \mapsto x_{n}+M_{k}\left(I_{n}\right)
$$

of $M_{k}\left(A_{0}\right) / M_{k}\left(I_{n}^{\prime}\right)$ to $M_{k}\left(A_{n}\right) / M_{k}\left(I_{n}\right)$ induces an isometric isomorphism between the Haagerup tensor products of these quotients with themselves under which $u^{M_{k}\left(I_{n}^{\prime}\right)}$ is mapped to $\left(u_{n}\right)^{M_{k}\left(I_{n}\right)}$.

Now considering $G \in \operatorname{Glimm}\left(A_{0}\right)$ of the form $G=G_{n}^{\prime}$ (some $n \geq 1$ and $G_{n} \in$ $\left.\operatorname{Glimm}\left(A_{n}\right)\right)$, we have

$$
\begin{aligned}
\left\|u^{M_{k}(G)}\right\|_{h} & =\left\|\left(u_{n}\right)^{M_{k}\left(G_{n}\right)}\right\|_{h} \leq L\left(M_{k}\left(A_{n}\right)\right)\left\|\theta_{A_{n}}\left(u_{n}\right)\right\|_{c b} \\
& \leq L_{k} \sup \left\{\left\|\left(u_{n}\right)^{M_{k}\left(P_{n}\right)}\right\|_{h}: P_{n} \in \operatorname{Prim}\left(A_{n}\right)\right\} \\
& \leq L_{k} \sup \left\{\left\|u^{M_{k}(P)}\right\|_{h}: P \in \operatorname{Prim}(A)\right\} \\
& =L_{k}\|\theta(u)\|_{c b} .
\end{aligned}
$$

Hence

$$
\|u\|_{Z, h}=\sup \left\{\left\|u^{M_{k}(G)}\right\|_{h}: G \in \operatorname{Glimm}(A)\right\} \leq L_{k}\|\theta(u)\|_{c b}
$$

and $L\left(M_{k}\left(A_{0}\right)\right) \leq L_{k}$.

Notation 4.6. By an (abstract finite) simplicial complex we understand a collection $\mathcal{C}$ of subsets of $\{1,2, \ldots, N\}$ closed under taking subsets $(E \in \mathcal{C}, F \subseteq E$ implies $F \in \mathcal{C})$ and containing all singletons $(\{i\} \in \mathcal{C}$ for $1 \leq i \leq N)$. We refer to $N$ as the number of vertices in $\mathcal{C}$. All complexes we deal with will be connected (given two vertices $i, j, 1 \leq i, j \leq N$, there is a sequence $i_{0}=i, i_{1}, \ldots, i_{m}=j$ so that $\left\{i_{k-1}, i_{k}\right\} \in \mathcal{C}$ for $\left.1 \leq k \leq m\right)$.

For subsets $E \subseteq\{1,2, \ldots, N\}$ we treat $\mathbb{C}^{E}$ (the set of functions from $E$ to $\mathbb{C}$ ) as a subspace of $\mathbb{C}^{N}$, namely the subspace spanned by those standard basis vectors $e_{i}$ of $\mathbb{C}^{N}$ with $i \in E$. (This will be more convenient for us than identifying $\mathbb{C}^{E}$ with $\mathbb{C}^{|E|}$.) We treat $\mathbb{C}^{E}$ as a commutative $C^{*}$-algebra with pointwise operations and the supremum norm. We will also use $M_{E}$ for matrices of size $|E| \times|E|$ indexed by $i, j \in E$ (with the operator norm from action on $\ell^{2}(E)$ ). Given a function $y=\left(y_{i}\right)_{i \in E} \in \mathbb{C}^{E}$, we let $\operatorname{diag}_{E}(y)$ be the diagonal matrix in $M_{E}$ with its $(i, i)$ entry equal to $y_{i}$ for each $i \in E$. Let $P_{E}: \mathbb{C}^{N} \rightarrow \mathbb{C}^{E}$ denote the projection (restriction) $P_{E}(y)=\left(y_{j}\right)_{j \in E}$.

We now define a 'simplicial spoke algebra' $A(\mathcal{C})$ associated to a connected simplicial complex as follows. Enumerate the maximal elements of $\mathcal{C}$ as $E_{1}, E_{2}, \ldots, E_{m}$ (maximal under set inclusion). Choose $m$ disjoint half-closed rays $R_{r}(1 \leq r \leq m)$ in the plane (say $R_{r}=\left\{t \zeta_{r}: t \geq 1\right\}$, where $\zeta_{1}, \zeta_{2}, \ldots, \zeta_{m}$ are distinct points of the unit circle). Let $\mathcal{T}=\bigcup_{r=1}^{m} R_{r}$.

We construct the algebra $A(\mathcal{C})$ as the subset of the functions

$$
x: \mathcal{T} \rightarrow \bigcup_{r=1}^{m} M_{E_{r}}(\mathbb{C})
$$


satisfying

- for $\tau \in R_{r} \subset \mathcal{T}, x(\tau) \in M_{E_{r}}$,

- the restriction of $x$ to $R_{r}$ is a continuous function on $R_{r}$ with values in $M_{E_{r}}$

- there are scalars $\lambda_{i}(x)(1 \leq i \leq N)$ so that the limit as $\tau \in R_{r}$ tends to infinity of $x(\tau)$ is the diagonal matrix $\operatorname{diag}_{E}\left(\left(\lambda_{j}(x)\right)_{j \in E}\right)$.

By a 'constant' element of $A(\mathcal{C})$ we mean an element where $x$ is constant along $R_{r}$ for each $1 \leq r \leq m$. Such an element is determined by $\lambda_{i}(x)(1 \leq i \leq N)$. More specifically, given $y \in \mathbb{C}^{N}$ we define $x=\operatorname{const}(y)$ to be the element where $x(\tau)=$ $\operatorname{diag}_{E_{r}}\left(P_{E_{r}}(y)\right)$ when $\tau \in R_{r}$. The map $\lambda: A(\mathcal{C}) \rightarrow \mathbb{C}^{N}$ with $\lambda(x)=\left(\lambda_{i}(x)\right)_{i=1}^{N}$ is a left inverse for const; that is, $\lambda \circ$ const is the identity.

With pointwise operations and the supremum norm $\|x\|=\sup _{\tau \in \mathcal{T}}\|x(\tau)\|, A(\mathcal{C})$ is a unital $C^{*}$-algebra. It has a centre consisting of those $x$ where $x(\tau)$ is a multiple of the identity matrix (of the appropriate size) for each $\tau \in \mathcal{T}$. Thus, since $\mathcal{C}$ is connected, the centre can be identified with the continuous functions on the one-point compactification of $\mathcal{T}$.

$A(\mathcal{C})$ has one Glimm ideal $G_{\infty}$ that is not primitive (if $N>1$ ). The minimal primal ideals containing $G_{\infty}$ are the intersections $\bigcap\left\{\operatorname{ker} \lambda_{i}: i \in E_{r}\right\}(1 \leq r \leq m)$. Up to ${ }^{*}$-isomorphism, $A(\mathcal{C})$ is independendent of the enumeration $E_{1}, E_{2}, \ldots, E_{m}$ and the choice of $\zeta_{1}, \zeta_{2}, \ldots, \zeta_{m}$. Thus $L(A(\mathcal{C}))$ and $L^{\prime}(A(\mathcal{C}))$ depend only on $\mathcal{C}$. Note that the smallest possible (connected) $\mathcal{C}$ in cases $N=1$ and $N=2$ are exceptional. If $N=1$, then $A(\mathcal{C})$ is abelian, and if $N=2$ we must have $\{1,2\} \in \mathcal{C}$ so that $m=1$ and every Glimm ideal of $A(\mathcal{C})$ is primal. So in both these cases $L(A(\mathcal{C}))=L^{\prime}(A(\mathcal{C}))=1($ see Remark 4.2) .

This notation and construction generalises the construction in [10, §2]. The simplest example of interest occurs when $N=3$ and the maximal elements of $\mathcal{C}$ are $E_{1}=\{1,2\}, E_{2}=\{3,2\}$ and $E_{3}=\{1,2\}$. In this case, the algebra $A(\mathcal{C})$ has the essential features of the sequence algebra of [4, Example 4.12], with the three rays $R_{1}, R_{2}$ and $R_{3}$ replacing the use of three subsequences.

Lemma 4.7. For $A=A(\mathcal{C})$ a simplicial spoke algebra,

$$
L(A(\mathcal{C}))=\inf \left\{C>0:\|v\|_{h} \leq C \sup _{E \in \mathcal{C}}\left\|v_{E}\right\|_{h} \forall v \in \mathbb{C}^{N} \otimes \mathbb{C}^{N}\right\},
$$

where $N$ is the number of vertices in $\mathcal{C}, \mathbb{C}^{N}$ is the $N$-dimensional commutative $C^{*}$-algebra, and for $E \subseteq\{1,2, \ldots, N\}$ we use $v_{E}$ to denote the projection $\left(P_{E} \otimes P_{E}\right)(v) \in \mathbb{C}^{E} \otimes \mathbb{C}^{E}$.

Moreover, for each $n \geq 1$,

$$
L\left(M_{n}(A(\mathcal{C}))\right)=\inf \left\{C>0:\|v\|_{h} \leq C \sup _{E \in \mathcal{C}}\left\|v_{E}\right\|_{h} \forall v \in M_{n}\left(\mathbb{C}^{N}\right) \otimes M_{n}\left(\mathbb{C}^{N}\right)\right\}
$$

where now $v_{E}=\left(P_{E}^{(n)} \otimes P_{E}^{(n)}\right)(v) \in M_{n}\left(\mathbb{C}^{E}\right) \otimes M_{n}\left(\mathbb{C}^{E}\right)$.

Proof. Fix $n \geq 1$. To fix the notation, we take $M_{n}(X)$ to mean $X \otimes M_{n}$, and the ampliation $\phi^{(n)}$ of a map $\phi: X \rightarrow Y$ to be $\phi \otimes \operatorname{id}_{n}$.

For $v=\sum_{j=1}^{\ell} a_{j} \otimes b_{j} \in M_{n}\left(\mathbb{C}^{N}\right) \otimes M_{n}\left(\mathbb{C}^{N}\right)$, we can construct

$$
u=\sum_{j=1}^{\ell} \operatorname{const}^{(n)}\left(a_{j}\right) \otimes \operatorname{const}^{(n)}\left(b_{j}\right) \in M_{n}(A(\mathcal{C})) \otimes M_{n}(A(\mathcal{C})) .
$$


We note that the map const: $\mathbb{C}^{N} \rightarrow A(\mathcal{C})$ is an embedding of $C^{*}$-algebras, hence a complete isometry to which we can apply Lemma 2.3 .

From Theorem 3.5 (or [22, Theorem 1] since we are in a unital algebra), we have

$$
\|u\|_{Z, h}=\sup \left\{\left\|u^{M_{n}(G)}\right\|_{h}: G \in \operatorname{Glimm}(A(\mathcal{C}))\right\},
$$

and by Proposition 3.6

$$
\left\|\theta_{Z}(u)\right\|_{c b}=\sup \left\{\left\|u^{M_{n}(P)}\right\|_{h}: P \in \operatorname{Prim}(A(\mathcal{C}))\right\} .
$$

We know that $\operatorname{Prim}(A(\mathcal{C}))$ consists of the kernels of representations $\pi_{\tau}: A(\mathcal{C}) \rightarrow$ $M_{E_{r}}: x \mapsto x(\tau)$ with $\tau \in R_{r}, 1 \leq r \leq m$, together with the kernels of the 1dimensional representations $\lambda_{i}(1 \leq i \leq N)$. For $P=\operatorname{ker} \pi_{\tau}$ we know that the quotient $A(\mathcal{C}) / P$ is $*$-algebra isomorphic to $M_{E_{r}}$, via $\bar{\pi}_{\tau}(x+P)=\pi_{\tau}(x)$. We can compute

$$
\begin{aligned}
\left(\bar{\pi}_{\tau}^{(n)} \otimes \bar{\pi}_{\tau}^{(n)}\right)\left(u^{M_{n}(P)}\right) & \\
\quad= & \sum_{j=1}^{\ell} \bar{\pi}_{\tau}^{(n)}\left(\operatorname{const}^{(n)}\left(a_{j}\right)+M_{n}(P)\right) \otimes \bar{\pi}_{\tau}^{(n)}\left(\operatorname{const}^{(n)}\left(b_{j}\right)+M_{n}(P)\right) \\
& =\sum_{j=1}^{\ell} \operatorname{diag}_{E_{r}}^{(n)}\left(P_{E_{r}}^{(n)}\left(a_{j}\right)\right) \otimes \operatorname{diag}_{E_{r}}^{(n)}\left(P_{E_{r}}^{(n)}\left(b_{j}\right)\right) \\
& =\left(\operatorname{diag}_{E_{r}}^{(n)} \otimes \operatorname{diag}_{E_{r}}^{(n)}\right) v_{E_{r}} .
\end{aligned}
$$

Thus $\left\|u^{M_{n}(P)}\right\|_{h}=\left\|v_{E_{r}}\right\|_{h}$.

On the other hand, for $P=\operatorname{ker} \lambda_{i}$ there is at least one $r$ so that $\{i\} \subseteq E_{r}$ and then $\left\|u^{M_{n}(P)}\right\|_{h}=\left\|v_{\{i\}}\right\|_{h} \leq\left\|v_{E_{r}}\right\|_{h}$ (for example, by applying Lemma 2.3 to the projection of $\mathbb{C}^{E_{r}}$ onto one coordinate). Hence

$$
\begin{aligned}
\left\|\theta_{Z}(u)\right\|_{c b} & =\sup _{P \in \operatorname{Prim}(A(\mathcal{C}))}\left\|u^{M_{n}(P)}\right\|_{h}=\sup _{1 \leq r \leq m}\left\|v_{E_{r}}\right\|_{h} \\
& =\sup \left\{\left\|v_{E}\right\|_{h}: E \in \mathcal{C}\right\} .
\end{aligned}
$$

(Here we can use Lemma2.3 to justify the last equality. If $E \subseteq E_{r}$, then the orthogonal projection $P_{E}$ factors through $P_{E_{r}}$, and these projections are homomorphisms of commutative $C^{*}$-algebras.)

For $G=G_{\infty}$, we have $A(\mathcal{C}) / G$ isomorphic to $\mathbb{C}^{N}$ and $\left\|u^{M_{n}(G)}\right\|_{h}=\|v\|_{h}$. To verify this, let $\bar{\lambda}: A(\mathcal{C}) / G_{\infty} \rightarrow \mathbb{C}^{N}$ given by $\bar{\lambda}\left(x+G_{\infty}\right)=\lambda(x)$ be the canonical isomorphism. Let $q: A(\mathcal{C}) \rightarrow A(\mathcal{C}) / G_{\infty}$ be the quotient map and observe that $\bar{\lambda} \circ q \circ$ const $=\lambda \circ$ const is the identity on $\mathbb{C}^{N}$. Thus

$$
v=(\bar{\lambda} \circ q \circ \text { const })^{(n)} \otimes(\bar{\lambda} \circ q \circ \text { const })^{(n)}(v)=\left(\bar{\lambda}^{(n)} \otimes \bar{\lambda}^{(n)}\right) u^{M_{n}\left(G_{\infty}\right)},
$$

and $\left\|u^{M_{n}(G)}\right\|_{h}=\|v\|_{h}$ follows by Lemma 2.3. For other $G \in \operatorname{Glimm}(A(\mathcal{C}))$ we have $G=\operatorname{ker} \pi_{\tau}$ (some $\tau \in R_{r} \subseteq \mathcal{T}, 1 \leq r \leq m$ ) primitive, and in this case we have shown earlier that $\left\|u^{M_{n}(G)}\right\|_{h}=\left\|v_{E_{r}}\right\|_{h} \leq\|v\|_{h}$. Thus

$$
\|u\|_{Z, h}=\sup _{G \in \operatorname{Glimm}(A(\mathcal{C}))}\left\|u^{M_{n}(G)}\right\|_{h}=\|v\|_{h} .
$$

We can now deduce from (4.4) and (4.5) that $L\left(M_{n}(A(\mathcal{C}))\right) \geq L_{n}(\mathcal{C})$, where

$$
L_{n}(\mathcal{C})=\inf \left\{C>0:\|v\|_{h} \leq C \sup _{E \in \mathcal{C}}\left\|v_{E}\right\|_{h} \forall v \in M_{n}\left(\mathbb{C}^{N}\right) \otimes M_{n}\left(\mathbb{C}^{N}\right)\right\} .
$$


To show $L\left(M_{n}(A(\mathcal{C}))\right) \leq L_{n}(\mathcal{C})$, consider an arbitrary $u \in M_{n}(A(\mathcal{C})) \otimes M_{n}(A(\mathcal{C}))$. From $u^{M_{n}\left(G_{\infty}\right)}$ and the isomorphism $\bar{\lambda}: A(\mathcal{C}) / G_{\infty} \rightarrow \mathbb{C}^{N}$ we get

$$
v=\left(\bar{\lambda}^{(n)} \otimes \bar{\lambda}^{(n)}\right)\left(u^{M_{n}\left(G_{\infty}\right)}\right)=\left(\lambda^{(n)} \otimes \lambda^{(n)}\right)(u) \in M_{n}\left(\mathbb{C}^{N}\right) \otimes M_{n}\left(\mathbb{C}^{N}\right) .
$$

Then we have $\left\|u^{M_{n}\left(G_{\infty}\right)}\right\|_{h}=\|v\|_{h}$ because $\bar{\lambda}$ is an isomorphism of $C^{*}$-algebras.

By Proposition 3.7 (or [22, Proposition 3]) we know $\left\|\theta_{Z}(u)\right\|_{c b}=\sup \left\{\left\|u^{M_{n}(Q)}\right\|_{h}\right.$ : $Q \in \operatorname{Primal}(A(\mathcal{C}))\}$. Since the primal ideals of $A(\mathcal{C})$ which contain $G_{\infty}$ are those of the form $Q=\bigcap_{i \in E} \operatorname{ker} \lambda_{i}$ for $E \in \mathcal{C}$, and $x+Q \mapsto \bar{\lambda}_{E}(x+Q):=P_{E}(\lambda(x))$ is an isomorphism $A / Q \rightarrow \mathbb{C}^{E}$, it follows that $\left\|u^{M_{n}(Q)}\right\|_{h}=\left\|\left(\bar{\lambda}_{E}^{(n)} \otimes \bar{\lambda}_{E}^{(n)}\right)\left(u^{M_{n}(Q)}\right)\right\|_{h}=$ $\left\|\left(P_{E}^{(n)} \otimes P_{E}^{(n)}\right)\left(\lambda^{(n)} \otimes \lambda^{(n)}\right)(u)\right\|_{h}=\left\|v_{E}\right\|$. Thus

$$
\left\|\theta_{Z}(u)\right\|_{c b} \geq \sup \left\{\left\|v_{E}\right\|_{h}: E \in \mathcal{C}\right\}
$$

We deduce

$$
\left\|u^{M_{N}\left(G_{\infty}\right)}\right\|_{h}=\|v\|_{h} \leq L_{n}(\mathcal{C})\left\|\theta_{Z}(u)\right\|_{c b} .
$$

As $L_{n}(\mathcal{C}) \geq 1$ and all other Glimm ideals of $A(\mathcal{C})$ are primitive (and primal), we have

$$
\|u\|_{Z, h}=\sup \left\{\left\|u^{M_{n}(G)}\right\|_{h}: G \in \operatorname{Glimm}(A(\mathcal{C}))\right\} \leq L_{n}(\mathcal{C})\left\|\theta_{Z}(u)\right\|_{c b} .
$$

This establishes $L\left(M_{n}(A(\mathcal{C}))\right) \leq L_{n}(\mathcal{C})$, and so equality, as claimed.

Remarks 4.8. (a) The first assertion of Lemma 4.7 may be restated in terms of norms of Schur multipliers. For $v \in \mathbb{C}^{N} \otimes \mathbb{C}^{N}$, we can compute $\|v\|_{h}$ via Haagerup's theorem as a cb norm. We embed $\mathbb{C}^{N}$ as the diagonal in $M_{N}$ and then $\|v\|_{h}=\left\|\left(\operatorname{diag}_{N} \otimes \operatorname{diag}_{N}\right)(v)\right\|_{h}=\left\|\theta\left(\left(\operatorname{diag}_{N} \otimes \operatorname{diag}_{N}\right)(v)\right)\right\|_{c b}$. Note that the operator $T=\theta\left(\left(\operatorname{diag}_{N} \otimes \operatorname{diag}_{N}\right)(v)\right)$ on $M_{N}$ is a Schur multiplier and that it is of the form $T(x)=T\left(\left(x_{i j}\right)_{i, j=1}^{N}\right)=\left(\gamma_{i j} x_{i j}\right)_{i, j=1}^{N}$, and so $\|T\|_{c b}=\|T\|\left(\left[19\right.\right.$, Theorem 8.7]). Moreover all Schur multipliers on $M_{N}$ arise from tensors $v \in \mathbb{C}^{N} \otimes \mathbb{C}^{N}$ in this way.

As $\left\|v_{E}\right\|_{h}$ for $E \subset\{1,2, \ldots, N\}$ is the norm of the Schur multiplier on $M_{E}$ which arises by taking those $\gamma_{i j}$ where $i, j \in E$, the computation of $L(A(\mathcal{C}))$ is then equivalent to finding the maximum possible norm of a Schur multiplier on $M_{N}$ given by $\gamma=\left(\gamma_{i j}\right)_{i, j=1}^{N}$ under the restriction that certain submultipliers (arising by taking $i, j \in E, E \in \mathcal{C}$ ) have norm at most 1 .

(b) One can then see that if there exists a pair $\left(i_{0}, j_{0}\right)$ where $\left\{i_{0}, j_{0}\right\}$ is not contained in any $E \in \mathcal{C}$, then the choice of $\gamma$ with an arbitrary entry in the $\left(i_{0}, j_{0}\right)$ position (and zeroes elsewhere) shows that the maximum is unbounded. In this case the intersection $\operatorname{ker} \lambda_{i_{0}} \cap \operatorname{ker} \lambda_{j_{0}}$ is not primal and $G_{\infty}$ is a Glimm ideal in $A(\mathcal{C})$ that fails to be 2-primal.

(c) Another observation is that given $\mathcal{C}$, if we add some more subsets of $\{1,2$, $\ldots, N\}$ to get a simplex $\mathcal{C}^{\prime} \supseteq \mathcal{C}$, then $L\left(M_{n}(A(\mathcal{C}))\right) \geq L\left(M_{n}\left(A\left(\mathcal{C}^{\prime}\right)\right)\right)$ by Lemma 4.7 .

\section{LOWER BOUNDS}

Let $A$ be a $C^{*}$-algebra and suppose $G \in \operatorname{Glimm}(A)$ is 2-primal. A finite subset $\left\{P_{1}, P_{2}, \ldots, P_{N}\right\} \subseteq \operatorname{Prim}(A / G)$ is called 'admissible' if $P_{i} \nsubseteq P_{j}$ for $i \neq j$. Associated with any such admissible set, we define a connected simplicial complex by $\mathcal{C}=\left\{E \subset\{1,2, \ldots, N\}: \bigcap_{i \in E} P_{i} \in \operatorname{Primal}(A)\right\}$. We say that such a simplicial complex is linked to the ideal structure of $A$. 
Theorem 5.1. Let $A$ be a $C^{*}$-algebra and $\mathcal{C}$ a simplicial complex linked to the ideal structure of $A$. Then $L\left(M_{n}(A)\right) \geq L\left(M_{n}(A(\mathcal{C}))\right)$ for $n \geq 1$ and $L^{\prime}(A) \geq L^{\prime}(A(\mathcal{C}))$.

The proof is an adaptation of the argument for [10, Theorem 7].

Proof. There is a 2-primal $G \in \operatorname{Glimm}(A)$ and an admissible subset

$$
\left\{P_{1}, P_{2}, \ldots, P_{N}\right\} \subseteq \operatorname{Prim}(A / G)
$$

such that $\mathcal{C}$ is the associated simplicial complex. Note that as $G$ is 2-primal, every 2-element set $\{i, j\} \in \mathcal{C}(1 \leq i<j \leq N)$ and so $\mathcal{C}$ is certainly connected. Let $J:=\bigcap_{j=1}^{N} P_{j}$.

For each subset $E \subset\{1,2, \ldots, N\}$ that fails to belong to $\mathcal{C}$ there must exist open neighbourhoods $U_{j, E}$ of each $P_{j}$ with $j \in E$ that satisfy $\bigcap_{j \in E} U_{j, E}=\emptyset$. For, if no such neighbourhoods existed there would be a net $\left(Q_{\alpha}\right)_{\alpha}$ in $\operatorname{Prim}(A)$ converging to each of the $P_{j}$ with $j \in E$ - showing $\bigcap_{i \in E} P_{i}$ primal and contradicting $E \notin \mathcal{C}$ [5, Proposition 3.2]. Let $U_{i}=\bigcap_{E \ni i, E \notin \mathcal{C}} U_{i, E}$, for $1 \leq i \leq N$. Note that each $U_{i}$ is an open neighbourhood of $P_{i}$ and that for each $E \notin \mathcal{C}$ we have $\bigcap_{i \in E} U_{i}=\emptyset$.

Now there are closed two-sided ideals $J_{i}$ in $A$ so that $U_{i}=\operatorname{Prim}\left(J_{i}\right)$ (hence $\left.U_{i}=\left\{Q \in \operatorname{Prim}(A): J_{i} \not \subset Q\right\}\right)$. Let

$$
R_{j}:=\bigcap_{i \neq j} P_{i} \quad(1 \leq j \leq N) .
$$

Let $I_{j}=J_{j} R_{j}$ for each $j$. The ideal $I_{j}$ cannot be contained in $J$ because then we would have $J_{j} R_{j} \subseteq P_{j}$, and since the primitive ideal $P_{j}$ is necessarily prime, it would follow that $J_{j} \subseteq P_{j}$ or $R_{j} \subseteq P_{j}$. Since $P_{j} \in U_{j}$, we have $J_{j} \nsubseteq P_{j}$. By primeness of $P_{j}$, if $R_{j} \subseteq P_{j}$, then $P_{i} \subseteq P_{j}$ for some $i \neq j$ (again not so).

Let $\Psi: A \rightarrow A / J$ denote the quotient map. Let $K_{j}=\Psi\left(I_{j}\right)$, a non-zero closed ideal of $A / J$. Note that $K_{j} K_{k}=0$ for $j \neq k$ (as $R_{j} R_{k} \subseteq J$ ).

For $1 \leq j \leq N$, choose a positive element $d_{j} \in K_{j}$ of norm one and $g_{j} \in I_{j}$ positive of norm one with $\Psi\left(g_{j}\right)=d_{j}^{1 / 3}$. Since $d_{j}^{1 / 3} d_{k}^{1 / 3}=0$ for $j \neq k$, we can use 2. Proposition 2.6] to find $c_{j} \in A^{+}(1 \leq j \leq N)$ with $\Psi\left(c_{j}\right)=\Psi\left(g_{j}\right)=d_{j}^{1 / 3}$ and $c_{j} c_{k}=0$ for $j \neq k$. Let $b_{j}^{\prime}=c_{j} g_{j} c_{j} \in I_{j}^{+}$. Then $b_{j}^{\prime} b_{k}^{\prime}=0$ for $j \neq k$ and $\Psi\left(b_{j}^{\prime}\right)=d_{j}$ $(1 \leq j, k \leq N)$.

Let $f:[0, \infty) \rightarrow[0, \infty)$ be $f(t)=\min (t, 1)$, a uniform limit on any compact subset of $[0, \infty)$ of polynomials without constant term. Define $b_{j}=f\left(b_{j}^{\prime}\right)$ by functional calculus. Then we have $b_{j} \in I_{j}^{+}, \Psi\left(b_{j}\right)=d_{j},\left\|b_{j}\right\|=1$ and $b_{j} b_{k}=0$ for $j \neq k$.

Now, if $C<L\left(M_{n}(A(\mathcal{C}))\right)$, then by Lemma 4.7 there exists $v \in M_{n}\left(\mathbb{C}^{N}\right) \otimes$ $M_{n}\left(\mathbb{C}^{N}\right)$ with $\|v\|_{h}>C$ and $\sup _{E \in \mathcal{C}}\left\|v_{E}\right\|_{h} \leq 1$. Consider the map $\phi: \mathbb{C}^{N} \rightarrow A$ defined by $\phi\left(e_{j}\right)=b_{j}$. Put $u=\left(\phi^{(n)} \otimes \phi^{(n)}\right)(v)$. As the canonical quotient map from $M_{n}(A) / M_{n}(G)$ to $M_{n}(A) / M_{n}(J)$ induces a contraction from $M_{n}(A) / M_{n}(G) \otimes_{h}$ $M_{n}(A) / M_{n}(G)$ to $M_{n}(A) / M_{n}(J) \otimes_{h} M_{n}(A) / M_{n}(J)$, we have

$$
\|u\|_{Z, h} \geq\left\|u^{M_{n}(G)}\right\|_{h} \geq\left\|u^{M_{n}(J)}\right\|_{h}=\left\|\left(q_{J} \otimes q_{J}\right)(u)\right\|_{h},
$$

where $q_{J}: M_{n}(A) \rightarrow M_{n}(A) / M_{n}(J)$ is the quotient map.

Denote by $\Phi_{J}: M_{n}(A / J) \rightarrow M_{n}(A) / M_{n}(J)$ the natural identification map (a *-isomorphism). Let $Y=\operatorname{span}\left\{b_{1}+J, b_{2}+J, \ldots, b_{N}+J\right\}$ and $\psi: \mathbb{C}^{N} \rightarrow Y$ be the linear map with $\psi\left(e_{i}\right)=b_{i}+J$.

By Lemma 2.4 (iii),

$$
\|v\|_{h}=\left\|\left(\psi^{(n)} \otimes \psi^{(n)}\right)(v)\right\|_{h}
$$


(the Haagerup norm in $M_{n}(Y) \otimes M_{n}(Y)$, but that is the same as the Haagerup norm in $M_{n}(A / J) \otimes M_{n}(A / J)$ by injectivity of the Haagerup norm). Thus,

$$
\|v\|_{h}=\left\|\left(\psi^{(n)} \otimes \psi^{(n)}\right)(v)\right\|_{h}=\left\|\left(\Phi_{J} \otimes \Phi_{J}\right)\left(\psi^{(n)} \otimes \psi^{(n)}\right)(v)\right\|_{h} .
$$

One can check in a straightforward way that $\Phi_{J} \circ \psi^{(n)}=q_{J} \circ \phi^{(n)}$ (for example, by applying both sides to $a=e_{i} \otimes e_{j, k} \in M_{n}\left(\mathbb{C}^{N}\right)$ and using linearity), and so

$$
\begin{aligned}
\|v\|_{h} & =\left\|\left(\Phi_{J} \otimes \Phi_{J}\right)\left(\psi^{(n)} \otimes \psi^{(n)}\right)(v)\right\|_{h} \\
& =\left\|\left(q_{J} \otimes q_{J}\right)\left(\phi^{(n)} \otimes \phi^{(n)}\right)(v)\right\|_{h} \\
& =\left\|\left(q_{J} \otimes q_{J}\right)(u)\right\|_{h} \leq\|u\|_{Z, h} .
\end{aligned}
$$

We deduce $\|u\|_{Z, h}>C$.

On the other hand, if $P \in \operatorname{Prim}(A)$, we know $E=\left\{j: 1 \leq j \leq N, P \in U_{j}\right\} \in \mathcal{C}$, and so $b_{j}+P=0 \forall j \notin E$. We define $\phi_{E}: \mathbb{C}^{E} \rightarrow A / P$ by $\phi_{E}\left(e_{j}\right)=b_{j}+P$ for $j \in E$, let $q_{P}: M_{n}(A) \rightarrow M_{n}(A) / M_{n}(P)$ be the quotient map and let $\Phi_{P}: M_{n}(A / P) \rightarrow$ $M_{n}(A) / M_{n}(P)$ be the natural identification map. We have

$$
\left(q_{P} \otimes q_{P}\right)(u)=\left(q_{P} \otimes q_{P}\right)\left(\phi^{(n)} \otimes \phi^{(n)}\right)(u)=\left(\Phi_{P} \otimes \Phi_{P}\right)\left(\phi_{E}^{(n)} \otimes \phi_{E}^{(n)}\right)\left(v_{E}\right) .
$$

Applying Lemma 2.4 (ii), we have

$$
1 \geq\left\|v_{E}\right\|_{h} \geq\left\|\left(\phi_{E}^{(n)} \otimes \phi_{E}^{(n)}\right)\left(v_{E}\right)\right\|_{h}
$$

because $\left\|b_{j}+P\right\| \leq 1$ for $i \in E$. Hence

$$
\begin{aligned}
1 & \geq\left\|\left(\phi_{E}^{(n)} \otimes \phi_{E}^{(n)}\right)\left(v_{E}\right)\right\|_{h} \\
& =\left\|\left(\Phi_{P} \otimes \Phi_{P}\right)\left(\phi_{E}^{(n)} \otimes \phi_{E}^{(n)}\right)\left(v_{E}\right)\right\|_{h} \\
& =\left\|\left(q_{P} \otimes q_{P}\right)(u)\right\|_{h}=\left\|u^{M_{n}(P)}\right\|_{h} .
\end{aligned}
$$

Hence $\sup \left\{\left\|u^{P}\right\|_{h}: P \in \operatorname{Prim}(A)\right\} \leq 1$, and we must have $L\left(M_{n}(A)\right)>C$. As $C$ was arbitrary, we have $L\left(M_{n}(A)\right) \geq L\left(M_{n}(A(\mathcal{C}))\right)$, as required.

\section{UPPER BOUNDS}

In this section we establish one of our main results, namely that $L^{\prime}(A)$ is the supremum of the lower bounds given in Theorem 5.1 .

Theorem 6.1. Let $A$ be a $C^{*}$-algebra in which all Glimm ideals are 2-primal. Then

$$
L^{\prime}(A)=\sup _{\mathcal{C}} L^{\prime}(A(\mathcal{C}))
$$

where the supremum is over all simplicial complexes $\mathcal{C}$ that are linked to the ideal structure of $A$.

Proof. From Theorem 5.1 we have $L^{\prime}(A) \geq \sup _{\mathcal{C}} L^{\prime}(A(\mathcal{C}))$, and the issue is the reverse inequality $L\left(M_{n}(A)\right) \leq \sup _{\mathcal{C}} L^{\prime}(A(\mathcal{C})$ ) (all $n \geq 1$ ). Since the simplicial complexes linked to the ideal structure of $M_{n}(A)$ are the same as those linked to the ideal structure of $A$, it suffices to prove this for $n=1$.

Fix $T<L(A)$ and $u=\sum_{j=1}^{\ell} a_{j} \otimes b_{j} \in A \otimes A$ so that $\left\|\theta_{Z}(u)\right\|_{c b} \leq 1$ while $\|u\|_{Z, h}>T$. From Theorem 3.5, we have

$$
\|u\|_{Z, h}=\sup \left\{\left\|u^{G}\right\|_{h}: G \in \operatorname{Glimm}(A)\right\},
$$

and thus there is $G \in \operatorname{Glimm}(A)$ with $\left\|u^{G}\right\|_{h}>T$. We know

$$
\left\|u^{G}\right\|_{h}=\sup \operatorname{tgm}\left(\mathcal{Q}\left(\mathbf{a}^{*}, \phi\right), \mathcal{Q}(\mathbf{b}, \psi)\right)
$$


with the supremum over $\phi, \psi \in \operatorname{co}((A / G))$ (Lemma 2.1). Choose $\phi, \psi$, each convex combinations of the pure states $g_{1}, g_{2}, \ldots, g_{t} \in \mathbb{P}(A / G)$ with

$$
\operatorname{tgm}\left(\mathcal{Q}\left(\mathbf{a}^{*}, \phi\right), \mathcal{Q}(\mathbf{b}, \psi)\right)>T .
$$

Now take the kernels of the GNS representations $\pi_{g_{r}}$ associated to $g_{r}(1 \leq r \leq t)$ and let $P_{1}, P_{2}, \ldots, P_{N}$ be those that are minimal with respect to inclusion within this finite set of kernels.

Choose one irreducible representation $\pi_{i}: A \rightarrow \mathcal{B}\left(H_{i}\right)$ with $\operatorname{ker} \pi_{i}=P_{i}(1 \leq i \leq$ $N)$. If there is any $r$ where $\pi_{g_{r}}$ is not equivalent to any of $\pi_{1}, \pi_{2}, \ldots, \pi_{N}$, then we can choose some $i(1 \leq i \leq N)$ with $g_{r}\left(P_{i}\right)=0$. Then by [16, 3.4.2 (ii)], $g_{r}$ can be weak ${ }^{*}$-approximated by pure states $\left\langle\pi_{i}(\cdot) \xi, \xi\right\rangle$. It follows that we can maintain (6.1) and replace $\phi, \psi$ by states of the form

$$
\begin{aligned}
& \phi=\sum_{i=1}^{N} \sum_{s=1}^{n_{i}} \alpha_{i, s}\left\langle\pi_{i}(\cdot) \xi_{i, s}, \xi_{i, s}\right\rangle \\
& \psi=\sum_{i=1}^{N} \sum_{s=1}^{n_{i}} \beta_{i, s}\left\langle\pi_{i}(\cdot) \xi_{i, s}, \xi_{i, s}\right\rangle
\end{aligned}
$$

where $\alpha_{i, s} \geq 0, \sum_{i=1}^{N} \sum_{s=1}^{n_{i}} \alpha_{i, s}=1$, there are similar conditions on $\beta_{i, s}$ and $\xi_{i, s} \in H_{i}$ are unit vectors.

Let $F_{i}$ denote the span in $H_{i}$ of $\left\{\xi_{i, s}, \pi_{i}\left(a_{j}^{*}\right) \xi_{i, s}, \pi_{i}\left(b_{j}\right) \xi_{i, s}: 1 \leq j \leq \ell, 1 \leq s \leq n_{i}\right\}$, and we also use $F_{i}$ for the orthogonal projection : $H_{i} \rightarrow F_{i}$. Let $n$ be the least common multiple of $\operatorname{dim}\left(F_{i}\right)(1 \leq i \leq N)$, and for each $i$ fix a unitary isomorphism $\tilde{F}_{i}: F_{i} \rightarrow \ell_{\operatorname{dim} F_{i}}^{2}=\mathbb{C}^{\operatorname{dim} F_{i}}$. Let $\eta_{i, s}=\tilde{F}_{i}\left(\xi_{i, s}\right) \in \ell_{\operatorname{dim} F_{i}}^{2}$ which we also consider to be inside $\ell_{n}^{2}$ (so that $\eta_{i, s} \in \ell_{n}^{2}$ is a unit vector). Let $\omega_{i, s}$ be the vector state on $M_{n}=\mathcal{B}\left(\ell_{n}^{2}\right)$ corresponding to $\eta_{i, s}$.

Consider the completely positive map $C_{i}: A \rightarrow M_{n}$ given by

$$
C_{i}(x)=\left(\tilde{F}_{i} F_{i} \pi_{i}(x) F_{i} \tilde{F}_{i}^{*}\right)^{\left(k_{i}\right)}
$$

where $k_{i}=n / \operatorname{dim} F_{i}$ and the superscript denotes the ampliation $\left(C_{i}(x)\right.$ is a block diagonal matrix with the same block repeated $k_{i}$ times). Observe that

$$
\begin{aligned}
\omega_{i, s}\left(C_{i}\left(a_{j}\right) C_{i}\left(a_{k}\right)^{*}\right) & =\left\langle C_{i}\left(a_{k}^{*}\right) \eta_{i, s}, C_{i}\left(a_{j}^{*}\right) \eta_{i, s}\right\rangle \\
& =\left\langle\tilde{F}_{i} F_{i} \pi_{i}\left(a_{k}\right)^{*} F_{i} \tilde{F}_{i}^{*} \eta_{i, s}, \tilde{F}_{i} F_{i} \pi_{i}\left(a_{j}\right)^{*} F_{i} \tilde{F}_{i}^{*} \eta_{i, s}\right\rangle \\
& =\left\langle F_{i} \pi_{i}\left(a_{k}\right)^{*} F_{i} \xi_{i, s}, F_{i} \pi_{i}\left(a_{j}\right)^{*} F_{i} \xi_{i, s}\right\rangle \\
& =\left\langle F_{i} \pi_{i}\left(a_{k}\right)^{*} \xi_{i, s}, F_{i} \pi_{i}\left(a_{j}\right)^{*} \xi_{i, s}\right\rangle \\
& =\left\langle\pi_{i}\left(a_{k}\right)^{*} \xi_{i, s}, \pi_{i}\left(a_{j}\right)^{*} \xi_{i, s}\right\rangle \\
& =\left\langle\pi_{i}\left(a_{j} a_{k}^{*}\right) \xi_{i, s}, \xi_{i, s}\right\rangle .
\end{aligned}
$$

Similarly

$$
\omega_{i, s}\left(C_{i}\left(b_{j}\right)^{*} C_{i}\left(b_{k}\right)\right)=\left\langle\pi_{i}\left(b_{j}^{*} b_{k}\right) \xi_{i, s}, \xi_{i, s}\right\rangle .
$$

Define a linear completely positive map $\alpha: A \rightarrow M_{n}\left(\mathbb{C}^{N}\right)=\mathbb{C}^{N} \otimes M_{n}$ by

$$
\alpha(x)=\sum_{i=1}^{N} e_{i} \otimes C_{i}(x)
$$


and let $w \in M_{n}\left(\mathbb{C}^{N}\right) \otimes M_{n}\left(\mathbb{C}^{N}\right)$ be given by $w=(\alpha \otimes \alpha)(u)=\sum_{j=1}^{\ell} \alpha\left(a_{j}\right) \otimes$ $\alpha\left(b_{j}\right)=\sum_{j=1}^{\ell} c_{j} \otimes d_{j}$ (say). To show $\|w\|_{h}>T$ we consider the two states $\phi^{\prime}, \psi^{\prime} \in$ $\mathbb{S}\left(M_{n}\left(\mathbb{C}^{N}\right)\right)$ given by

$$
\begin{aligned}
\phi^{\prime}\left(\sum_{i=1}^{N} e_{i} \otimes x_{i}\right) & =\sum_{i=1}^{N} \sum_{s=1}^{n_{i}} \alpha_{i, s} \omega_{i, s}\left(x_{i}\right), \\
\psi^{\prime}\left(\sum_{i=1}^{N} e_{i} \otimes x_{i}\right) & =\sum_{i=1}^{N} \sum_{s=1}^{n_{i}} \beta_{i, s} \omega_{i, s}\left(x_{i}\right) .
\end{aligned}
$$

Using (6.2) we can verify

$$
\phi^{\prime}\left(c_{j} c_{k}^{*}\right)=\phi^{\prime}\left(\alpha\left(a_{j}\right) \alpha\left(a_{k}\right)^{*}\right)=\phi^{\prime}\left(\sum_{i=1}^{N} e_{i} \otimes C_{i}\left(a_{j}\right) C_{i}\left(a_{k}\right)^{*}\right)=\phi\left(a_{j} a_{k}^{*}\right)
$$

and, using (6.3), $\psi^{\prime}\left(d_{j}^{*} d_{k}\right)=\psi\left(b_{j}^{*} b_{k}\right)$. It then follows that

$$
\operatorname{tgm}\left(\mathcal{Q}\left(\mathbf{c}^{*}, \phi^{\prime}\right), \mathcal{Q}\left(\mathbf{d}, \psi^{\prime}\right)\right)=\operatorname{tgm}\left(\mathcal{Q}\left(\mathbf{a}^{*}, \phi\right), \mathcal{Q}(\mathbf{b}, \psi)\right)>T
$$

and hence $\|w\|_{h}>T$.

Now consider the simplicial complex (linked to the ideal structure of $A$ ) $\mathcal{C}=\{E \subset$ $\left.\{1,2, \ldots, N\}: \bigcap_{i \in E} P_{i} \in \operatorname{Primal}(A)\right\}$. Our aim is to verify that $L\left(M_{n}(A(\mathcal{C}))\right)>T$ by use of Lemma 4.7 .

For $E \in \mathcal{C}$, we consider $w_{E} \in M_{n}\left(\mathbb{C}^{E}\right) \otimes M_{n}\left(\mathbb{C}^{E}\right)$, which we write as

$$
w_{E}=\left(P_{E}^{(n)} \otimes P_{E}^{(n)}\right)(w)=\sum_{j=1}^{\ell} P_{E}^{(n)}\left(c_{j}\right) \otimes P_{E}^{(n)}\left(d_{j}\right)=\sum_{j=1}^{\ell} c_{E j} \otimes d_{E j} .
$$

We rely on the fact that (by Lemma 2.1)

$$
\left\|w_{E}\right\|_{h}=\sup \operatorname{tgm}\left(\mathcal{Q}\left(\mathbf{c}_{E}^{*}, \Phi^{\prime}\right), \mathcal{Q}\left(\mathbf{d}_{E}, \Psi^{\prime}\right)\right)
$$

where the supremum is over $\Phi^{\prime}, \Psi^{\prime} \in \mathbb{S}\left(M_{n}\left(\mathbb{C}^{E}\right)\right)$. Because of Lemma 4.7, we will be done if we show $\left\|w_{E}\right\|_{h} \leq 1$.

Therefore we consider arbitrary fixed specific $\Phi^{\prime}, \Psi^{\prime} \in \mathbb{S}\left(M_{n}\left(\mathbb{C}^{E}\right)=\mathbb{S}\left(\mathbb{C}^{E} \otimes M_{n}\right)\right.$. We can write $\Phi^{\prime}$ as a convex combination

$$
\Phi^{\prime}\left(\sum_{i \in E} e_{i} \otimes x_{i}\right)=\sum_{i \in E} \gamma_{i} \Phi_{i}\left(x_{i}\right)
$$

for $\Phi_{i} \in \mathbb{S}\left(M_{n}\right)$. We can then express each $\Phi_{i}$ as a convex combination of $n$ vector states $\Omega_{i, s}(x)=\left\langle x \zeta_{i, s}, \zeta_{i, s}\right\rangle$ to get

$$
\Phi^{\prime}\left(\sum_{i \in E} e_{i} \otimes x_{i}\right)=\sum_{i \in E} \sum_{s=1}^{n} \gamma_{i, s} \Omega_{i, s}\left(x_{i}\right)
$$

$\left(\gamma_{i, s} \geq 0, \sum_{i \in E} \sum_{s=1}^{n} \gamma_{i, s}=1\right)$. Let $K=\bigcap_{i \in E} P_{i} \in \operatorname{Primal}(A)$, and define a completely positive contraction $\alpha_{E}: A / K \rightarrow M_{n}\left(\mathbb{C}^{E}\right)$ by

$$
\alpha_{E}(x+K)=\sum_{i \in E} e_{i} \otimes C_{i}(x)=P_{E}^{(n)}(\alpha(x)) .
$$


Note then that $c_{E j}=P_{E}^{(n)} c_{j}=P_{E}^{(n)} \alpha\left(a_{j}\right)=\alpha_{E}\left(a_{j}+K\right)$, and similarly $d_{E j}=$ $\alpha_{E}\left(b_{j}+K\right)$. We now define a positive linear functional $\Phi$ on $A / K$ by $\Phi=\Phi^{\prime} \circ \alpha_{E}$. It clearly satisfies $\|\Phi\| \leq 1$ and we claim

$$
\mathcal{Q}\left(\mathbf{c}_{E}^{*}, \Phi^{\prime}\right)=\left(\Phi^{\prime}\left(\alpha_{E}\left(a_{j}+K\right) \alpha_{E}\left(a_{k}+K\right)^{*}\right)\right)_{j, k=1}^{\ell} \leq\left(\Phi\left(a_{j} a_{k}^{*}+K\right)\right)_{j, k=1}^{\ell}
$$

(an inequality of positive semidefinite $\ell \times \ell$ matrices). As convex combinations of positive semidefinite matrices are positive semidefinite, this claim reduces to showing

$$
\left(\left\langle\left(C_{i}\left(a_{j} a_{k}^{*}\right)-C_{i}\left(a_{j}\right) C_{i}\left(a_{k}\right)^{*}\right) \zeta_{i, s}, \zeta_{i, s}\right\rangle\right)_{j, k=1}^{\ell} \geq 0
$$

(for $i \in E, 1 \leq s \leq n$ ). If we let $\mu_{i, s}=\tilde{F}_{i}^{*} \zeta_{i, s} \in F_{i} \subset H_{i}$, then we have

$$
\begin{aligned}
& \left\langle\left(C_{i}\left(a_{j} a_{k}^{*}\right)-C_{i}\left(a_{j}\right) C_{i}\left(a_{k}\right)^{*}\right) \zeta_{i, s}, \zeta_{i, s}\right\rangle \\
& \quad=\left\langle\pi_{i}\left(a_{j} a_{k}^{*}\right) \mu_{i, s}-\pi_{i}\left(a_{j}\right) F_{i} \pi_{i}\left(a_{k}\right)^{*} \mu_{i, s}, \mu_{i, s}\right\rangle \\
& \quad=\left\langle\pi_{i}\left(a_{j}\right)\left(I_{i}-F_{i}\right) \pi_{i}\left(a_{k}\right)^{*} \mu_{i, s}, \mu_{i, s}\right\rangle \\
& =\left\langle\left(I_{i}-F_{i}\right) \pi_{i}\left(a_{k}\right)^{*} \mu_{i, s}, \pi_{i}\left(a_{j}\right)^{*} \mu_{i, s}\right\rangle \\
& =\left\langle\left(I_{i}-F_{i}\right) \pi_{i}\left(a_{k}\right)^{*} \mu_{i, s},\left(I_{i}-F_{i}\right) \pi_{i}\left(a_{j}\right)^{*} \mu_{i, s}\right\rangle
\end{aligned}
$$

(where $I_{i}$ is the identity operator in $H_{i}$ ). From this it is clear that the matrix with these entries is positive.

In a similar way we express $\Psi^{\prime}$ as a convex combination, and define $\Psi(x+K)=$ $\Psi^{\prime}\left(\alpha_{E}(x)\right)$. We get an inequality of positive semidefinite matrices again,

$$
\mathcal{Q}\left(\mathbf{d}_{E}, \Psi^{\prime}\right)=\left(\Psi^{\prime}\left(\alpha_{E}\left(b_{j}+K\right)^{*} \alpha_{E}\left(b_{k}+K\right)\right)\right)_{j, k=1}^{\ell} \leq\left(\Psi\left(b_{j}^{*} b_{k}+K\right)\right)_{j, k=1}^{\ell},
$$

and $\left\|\Psi^{\prime}\right\| \leq 1$.

Now we can use monotonicity of tgm to get

$$
\begin{aligned}
& \operatorname{tgm}\left(\mathcal{Q}\left(\mathbf{c}_{E}^{*}, \Phi^{\prime}\right), \mathcal{Q}\left(\mathbf{d}_{E}, \Psi^{\prime}\right)\right) \\
& \quad \leq \operatorname{tgm}\left(\left(\Phi\left(a_{j} a_{k}^{*}+K\right)\right)_{j, k=1}^{\ell},\left(\Psi\left(b_{j}^{*} b_{k}+K\right)\right)_{j, k=1}^{\ell}\right) \\
& \quad \leq \sqrt{\|\Phi\|\|\Psi\|\left\|u^{K}\right\| \leq 1 .}
\end{aligned}
$$

Hence, in view of (6.4), $\left\|w_{E}\right\| \leq 1 \forall E \in \mathcal{C}$. Since $\|w\|_{h}>T$, by Lemma 4.7, we have $L\left(M_{n}(A(\mathcal{C}))\right)>T$. As $T<L(A)$ is arbitrary, we have $L^{\prime}(A(\mathcal{C})) \geq L(A)$.

As a simple consequence of Theorem 6.1 we now obtain another of the main results of this paper.

Theorem 6.2. If $A$ and $B$ are $C^{*}$-algebra where $\operatorname{Prim}(A)$ and $\operatorname{Prim}(B)$ are homeomorphic, then $L^{\prime}(A)=L^{\prime}(B)$.

Proof. Let $\Phi: \operatorname{Prim}(A) \rightarrow \operatorname{Prim}(B)$ be a homeomorphism. The set $\operatorname{Glimm}(A)$ is defined in terms of the topology of $\operatorname{Prim}(A)$, and we can see immediately that $P_{1}, P_{2} \in \operatorname{Prim}(A)$ contain the same Glimm ideal of $A$ (that is, are not separated by functions in $\left.C_{b}(\operatorname{Prim}(A))\right)$ if and only if $\Phi\left(P_{1}\right)$ and $\Phi\left(P_{2}\right)$ contain the same Glimm ideal of $B$. Moreover $P_{1} \subseteq P_{2} \Longleftrightarrow P_{2}$ is in the closure of $\left\{P_{1}\right\} \Longleftrightarrow \Phi\left(P_{1}\right) \subseteq$ $\Phi\left(P_{2}\right)$.

It follows that $\left\{P_{1}, P_{2}, \ldots, P_{N}\right\}$ is an admissible subset of $\operatorname{Prim}(A)$ if and only if $\left\{\Phi\left(P_{1}\right), \Phi\left(P_{2}\right), \ldots, \Phi\left(P_{N}\right)\right\}$ is an admissible subset of $\operatorname{Prim}(B)$.

For a non-empty subset $E \subseteq\{1,2, \ldots, N\}$, we have $\bigcap_{i \in E} P_{i} \in \operatorname{Primal}(A)$ if and only if there is a net in $\operatorname{Prim}(A)$ convergent to each $P_{i}(i \in E)$. Applying $\Phi$ we 
see that the simplicial complexes linked to the ideal structure of $A$ are identical to those that are linked to $B$. The result follows from Theorem 6.1

Corollary 6.3. Let $A$ be a $C^{*}$-algebra. Then $L^{\prime}(A)=L(A \otimes \mathcal{K}(H)$ ) (for $H$ separable infinite dimensional).

Proof. Since $P \mapsto P \otimes \mathcal{K}(H)$ is a homeomorphism of $\operatorname{Prim}(A)$ onto $\operatorname{Prim}(A \otimes \mathcal{K}(H))$ (see remarks in the Introduction), it follows that the Glimm ideals of $A \otimes \mathcal{K}(H)$ are those of the form $\tilde{G}=\bigcap\{P \otimes \mathcal{K}(H): P \in \operatorname{Prim}(A / G)\}$, where $G \in \operatorname{Glimm}(A)$. Clearly $G \otimes \mathcal{K}(H) \subseteq \tilde{G}$. On the other hand any primitive ideal of $A \otimes \mathcal{K}(H)$ containing $G \otimes \mathcal{K}(H)$ has the form $P \otimes \mathcal{K}(H)$ for some $P \in \operatorname{Prim}(A)$. Let $a \in G$, $T \in \mathcal{K}(H)$ with $T \neq 0$, and let $\pi$ be an irreducible representation of $A$ with kernel $P$. Then $\pi(a) \otimes T=(\pi \otimes$ id $)(a \otimes T)=0$, and hence $G \subseteq P$. Thus $\tilde{G}=G \otimes \mathcal{K}(H)$.

An argument similar to the proof of Proposition 4.3 then shows $L\left(M_{n}(A)\right) \leq$ $L(A \otimes \mathcal{K}(H))$ for each $n$. Hence $L^{\prime}(A) \leq L(A \otimes \mathcal{K}(H))$. On the other hand, by Theorem 6.2, $L(A \otimes \mathcal{K}(H)) \leq L^{\prime}(A \otimes \mathcal{K}(H))=L^{\prime}(A)$.

Note that it follows that $L(A)=L^{\prime}(A)$ if $A$ is a stable $C^{*}$-algebra, but we will exhibit examples in Examples 7.12 (iii), Proposition 7.13 and Corollary 7.14 where $L(A) \neq L^{\prime}(A)$.

\section{EXAMPLES AND COMBINATORIAL BOUNDS}

In this section, we develop some bounds for $L(A)$ (and $L^{\prime}(A)$ ) in terms of explicit combinatorial properties of the primal ideals of $A$ containing individual Glimm ideals. In the light of Theorem 6.1, one particular aim is to find estimates for $L(A)$ and $L^{\prime}(A)$ in the case where $A$ is a simplicial spoke algebra $A(\mathcal{C})$. While exact values in the range $(1, \infty)$ seem challenging to establish, we give a family of examples for which $L(A)$ is very close to $\sqrt{n}$ but $L^{\prime}(A)=n-1(n \geq 3)$. It then follows that infinitely many values of $L(A)$ (and $L^{\prime}(A)$ ) do arise. We show in addition that these constants can be arbitrarily close to 1 (while not equal to 1 ). Even when all Glimm ideals of $A$ are 2-primal (so that $\theta_{Z}$ is injective) we can have $L(A)=\infty$.

Examples 7.1. (i) Fix $N>2$ and $2 \leq k<N$. Let $\mathcal{C}_{N, k}$ be the simplicial complex consisting of all subsets of $\{1,2, \ldots, N\}$ of cardinality at most $k$. Via the earlier remarks in Remarks 4.8 (a), we can give a lower bound for $L\left(A\left(\mathcal{C}_{N, k}\right)\right.$ ).

It is clear that the norm of a Schur multiplier operator $T: M_{N} \rightarrow M_{N}$ given by $T x=\left(\gamma_{i j} x_{i j}\right)_{i, j=1}^{N}$ is at least $\max _{i, j}\left|\gamma_{i j}\right|$ (by taking $x=e_{i, j}$ to have 1 in the $(i, j$ ) entry and zeros elsewhere).

To get an inequality in the opposite direction, note that $T=\theta\left(\sum_{i=1}^{N} a_{i} \otimes b_{i}\right)$, where $a_{i}=e_{i, i}$ and $b_{i}=\sum_{j=1}^{N} \gamma_{i j} e_{j, j}$. Then

$$
\begin{aligned}
\|T\| & =\|T\|_{c b}=\left\|\sum_{i=1}^{N} a_{i} \otimes b_{i}\right\|_{h} \\
& \leq\left(\left\|\sum_{i=1}^{N} a_{i} a_{i}^{*}\right\|\left\|\sum_{i=1}^{N} b_{i}^{*} b_{i}\right\|\right)^{1 / 2} \leq \sqrt{N} \max _{i, j}\left|\gamma_{i j}\right| .
\end{aligned}
$$

The constant $\sqrt{N}$ is optimal because we can take $\gamma_{i j}=\zeta^{i j}$, where $\zeta=\exp (2 \pi \iota / N)$ is a primitive $N$ th root of unity. For this choice, we can apply the multiplier to the 
unitary matrix $x=\left(\bar{\zeta}^{i j} / \sqrt{N}\right)_{i, j=1}^{N}$ and get

$$
T x=\left(\frac{1}{\sqrt{N}}\right)_{i, j=1}^{N}=\sqrt{N}\left(\frac{1}{N}\right)_{i, j=1}^{N}
$$

of norm $\sqrt{N}$ (as it is $\sqrt{N}$ times a projection).

If $E$ is a projection onto $k$ of the coordinates, then $E\left(\gamma_{i j}\right)_{i, j=1}^{N} E^{*}$ is a $k \times k$ matrix with unimodular entries. Hence it has a Schur multiplier norm at most $\sqrt{k}$.

By Lemma 4.7 this leads to the lower bound $L\left(A\left(\mathcal{C}_{N, k}\right)\right) \geq \sqrt{N / k}$.

If we modify the example to replace the diagonal entries $\gamma_{i i}$ by 0 , we can check that $\|T x\| \geq(N-1) / \sqrt{N}$ with the same $x$ (because the constant vectors are eigenvectors of $T x$ with this eigenvalue). The $k \times k$ multipliers $E\left(\gamma_{i j}\right)_{i, j=1}^{N} E^{*}$ now have norm at most $\sqrt{k-1}$. So, if $k \leq N / 2$ we get a better estimate, $L\left(A\left(\mathcal{C}_{N, k}\right)\right) \geq$ $(\sqrt{N}-1 / \sqrt{N}) / \sqrt{k-1}$.

(ii) Let $\mathcal{C}$ be an arbitrary simplicial complex on $\{1,2, \ldots, N\}$ which contains all 2 element subsets $\{i, j\}(1 \leq i, j \leq N)$ (so that the Glimm ideal $G_{\infty}$ is 2-primal). Then $L(A(\mathcal{C})) \leq \sqrt{N}$.

This inequality follows from Lemma 4.7. Remarks 4.8 (a) and the observations above about norms of Schur multipliers. If we consider a Schur multiplier $T$ with matrix $\left(\gamma_{i, j}\right)_{i, j=1}^{N}$ and $v \in \mathbb{C}^{N} \otimes \mathbb{C}^{N}$ as in Remarks 4.8 (a), then $\left|\gamma_{r, s}\right| \leq\left\|v_{E}\right\|_{h}$ for $r, s \in E$. Hence $\max _{i, j}\left|\gamma_{i, j}\right| \leq \max \left\{\left\|v_{E}\right\|_{h}: E \in \mathcal{C}\right\}$, and it follows that $\|v\|_{h}=\|T\|_{c b}=\|T\| \leq \sqrt{N} \max _{E}\left\|v_{E}\right\|_{h}$. Thus $L(A(\mathcal{C})) \leq \sqrt{N}$ by Lemma 4.7

(iii) From (i) and (ii) we now have examples such as $A=A\left(\mathcal{C}_{N, 2}\right)$, where $L(A)<$ $\infty$ and yet $L(A)$ can be arbitrarily large; we have $\sqrt{N / k} \leq L\left(A\left(\mathcal{C}_{N, k}\right)\right) \leq \sqrt{N}$ (for $2 \leq k<N)$ and $\sqrt{N}-1 / \sqrt{N} \leq L\left(A\left(\mathcal{C}_{N, 2}\right)\right) \leq \sqrt{N}$ for $N \geq 4$.

We show below (Theorem [7.10) that $L^{\prime}\left(A\left(\mathcal{C}_{N, 2}\right)\right)=N-1$, but our proof goes via identifying the best constant for a seemingly elementary inequality concerning operator norms of matrices.

Definition 7.2. For $\mathcal{C}$ a simplicial complex on $\{1,2, \ldots, N\}$, we define another constant $L_{\mathbb{C}}(\mathcal{C})$ to be the supremum

$$
L_{\mathbb{C}}(\mathcal{C})=\sup \left\{\|a\|: a=\left(a_{i, j}\right)_{i, j=1}^{N} \in M_{N}, \max _{E \in \mathcal{C}}\left\|a_{E}\right\| \leq 1\right\},
$$

where $a_{E}=\left(a_{i, j}\right)_{i, j \in E} \in M_{E}$.

It may be helpful to observe that in Examples 7.1 above, we are using the relationship between $L(A(\mathcal{C})$ ) and Schur multiplier norms (rather than operator norms) of matrices.

Our immediate goal is to compute $L_{\mathbb{C}}\left(\mathcal{C}_{N, 2}\right)=N-1$ and then to show in general that $L^{\prime}(A(\mathcal{C})) \leq L_{\mathbb{C}}(\mathcal{C})$. Finally, to establish $L^{\prime}\left(A\left(\left(\mathcal{C}_{N, 2}\right)\right)\right)=N-1$ we will need to construct a further example.

Lemma 7.3. Suppose $H, K$ are Hilbert spaces and we have orthogonal decompositions $H=H_{1} \oplus H_{2} \oplus \cdots \oplus H_{N}$ and $K=K_{1} \oplus K_{2} \oplus \cdots \oplus K_{N}$. Fix $1 \leq k \leq N$. For each subset $E \subset[N]=\{1,2, \ldots, N\}$ of cardinality $|E|=k$ suppose $x_{E} \in \mathcal{B}(H, K)$ is an operator of norm $\left\|x_{E}\right\| \leq 1$ satisfying

$$
\begin{aligned}
x_{E}\left(\bigoplus_{i \in E} H_{i}\right) & \subseteq \bigoplus_{i \in E} K_{i} \\
x_{E}\left(H_{j}\right) & =\{0\} \text { if } j \in[N] \backslash E .
\end{aligned}
$$


Then

$$
\left\|\sum_{E \subset[N],|E|=k} x_{E}\right\| \leq\left(\begin{array}{c}
N-1 \\
k-1
\end{array}\right)
$$

Note that the hypotheses may be stated as saying that $x_{E}$ has a block matrix $x_{E}=\left(x_{E, i, j}\right)_{i, j=1}^{N}$ with respect to the given decompositions of $H$ and $K$ that satisfies $x_{E, i, j}=0$ if either $i \notin E$ or $j \notin E$.

In the special case where $N=3, k=2$ and the subspaces $H_{i}$ and $K_{i}$ are 1 dimensional, we can state the conclusion as follows. If we have three $3 \times 3$ matrices of the form

$$
x=\left(\begin{array}{ccc}
0 & 0 & 0 \\
0 & x_{2,2} & x_{2,3} \\
0 & x_{3,2} & x_{3,3}
\end{array}\right), y=\left(\begin{array}{ccc}
y_{1,1} & 0 & y_{1,3} \\
0 & 0 & 0 \\
y_{3,1} & 0 & y_{3,3}
\end{array}\right), z=\left(\begin{array}{ccc}
z_{1,1} & z_{1,2} & 0 \\
z_{2,1} & z_{1,2} & 0 \\
0 & 0 & 0
\end{array}\right),
$$

then

$$
\|x+y+z\| \leq 2 \max (\|x\|,\|y\|,\|z\|) .
$$

Observe that if $\|x\|=\|y\|=\|z\|$, then this inequality improves on what the triangle inequality gives.

Proof of Lemma 7.3. Take a unit vector $\xi \in H$, which we write as $\xi=\sum_{i=1}^{N} \xi_{i}$ for $\xi_{i} \in H_{i}$. Write $\xi_{E}=\sum_{i \in E} \xi_{i}, \theta_{E}=x_{E} \xi$ (for $E \subset[N]$ with $|E|=k$ ), and note that as $\theta_{E}=x_{E} \xi_{E}$ we have $\left\|\theta_{E}\right\|^{2} \leq\left\|x_{E}\right\|^{2}\left\|\xi_{E}\right\|^{2} \leq \sum_{i \in E}\left|\xi_{i}\right|^{2}$. Thus

$$
\left\|\left(\sum_{E} x_{E}\right) \xi\right\|^{2}=\left\|\sum_{E} \theta_{E}\right\|^{2}=\sum_{i=1}^{N}\left\|\sum_{E \ni i} \theta_{E, i}\right\|^{2}
$$

where we write $\theta_{E}=\sum_{i \in E} \theta_{E, i}$ with $\theta_{E, i} \in K_{i}$. Thus using the triangle and Cauchy-Schwarz inequalities, and counting the number of $E$ with $i \in E$ for fixed $i$, we get

$$
\begin{aligned}
\left\|\left(\sum_{E} x_{E}\right) \xi\right\|^{2} & \leq \sum_{i=1}^{N}\left(\begin{array}{c}
N-1 \\
k-1
\end{array}\right) \sum_{E \ni i}\left\|\theta_{E, i}\right\|^{2} \\
& =\left(\begin{array}{c}
N-1 \\
k-1
\end{array}\right) \sum_{E} \sum_{i \in E}\left\|\theta_{E, i}\right\|^{2} \\
& =\left(\begin{array}{c}
N-1 \\
k-1
\end{array}\right) \sum_{E}\left\|\theta_{E}\right\|^{2} \\
& \leq\left(\begin{array}{c}
N-1 \\
k-1
\end{array}\right) \sum_{E}\left\|\xi_{E}\right\|^{2} \\
& =\left(\begin{array}{c}
N-1 \\
k-1
\end{array}\right) \sum_{i=1}^{N}\left\|\xi_{i}\right\|^{2}=\left(\begin{array}{c}
N-1 \\
k-1
\end{array}\right)^{2} .
\end{aligned}
$$

The result follows.

Lemma 7.4. Fix $0 \leq t \leq 1$ and $k \geq 2$. Let $T_{t}: M_{k} \rightarrow M_{k}$ be the Schur multiplication operator on $M_{k}$ given by the matrix $\gamma=\left(\gamma_{i, j}\right)_{i, j=1}^{k}$, where $\gamma_{i, j}=1$ for $i \neq j$ and $\gamma_{i, i}=t$. Then $\left\|T_{t}\right\| \leq 1+(1-t)(1-2 / k)$. 
Proof. When $t=0$, [10, Example 2] says $\left\|T_{0}\right\|=2(1-1 / k)$. For $t=1, T_{1}$ is the identity on $M_{k}$ and has norm 1 . As $T_{t}=(1-t) T_{0}+t T_{1}$ we have

$$
\left\|T_{t}\right\| \leq 2(1-t)(1-1 / k)+t=1+(1-t)(1-2 / k) .
$$

Proposition 7.5. For $2 \leq k<N, L_{\mathbb{C}}\left(\mathcal{C}_{N, k}\right)=\frac{2 N}{k}-1$.

Proof. Consider a matrix $a=\left(a_{i, j}\right)_{i, j=1}^{N}$ (with complex entries $a_{i, j} \in \mathbb{C}$ ) satisfying $\sup _{E \in \mathcal{C}_{N, k}}\left\|a_{E}\right\| \leq 1$.

Let $b=\left(b_{i, j}\right)_{i, j=1}^{N}$ be defined by $b_{i, i}=((k-1) /(N-1)) a_{i, i}$ and $b_{i, j}=a_{i, j}$ for $i \neq j$. From Lemma 7.4 with $t=(k-1) /(N-1), 1-t=(N-k) /(N-1)$, we have that, for $|E|=k,\left\|b_{E}\right\| \leq 1+(1-2 / k)(N-k) /(N-1)$.

By Lemma 7.3

$$
\begin{aligned}
\|a\| & =\| \frac{1}{\left(\begin{array}{l}
N-2 \\
k-2
\end{array}\right)} \sum_{|E|=k} b_{E} \mid \\
& \leq \frac{\left(\begin{array}{l}
N-1 \\
k-1
\end{array}\right)}{\left(\begin{array}{l}
N-2 \\
k-2
\end{array}\right)}\left(1+(1-2 / k) \frac{N-k}{N-1}\right) \\
& =\frac{N-1}{k-1}\left(1+(1-2 / k) \frac{N-k}{N-1}\right)=2 \frac{N}{k}-1 .
\end{aligned}
$$

Hence $L_{\mathbb{C}}\left(\mathcal{C}_{N, k}\right) \leq \frac{2 N}{k}-1$.

To show equality, consider $a$, where $a_{i, i}=\frac{2}{k}-1$ and $a_{i, j}=2 / k$ for $i \neq j$. When $|E|=k, a_{E}$ is the $k \times k$ matrix $2 p_{k}-I_{k}$ with rows and columns of zeros added, where $p_{k} \in M_{k}$ is the rank one projection with entries $1 / k$. As $2 p_{k}-I_{k}=p_{k}-\left(I_{k}-p_{k}\right)$ is unitary, we have $\left\|a_{E}\right\|=1$ when $|E|=k$.

When we apply $a$ to the vector $\xi$ with all coordinates 1 we get

$$
a \xi=\left(\frac{2}{k}-1+(N-1) \frac{2}{k}\right) \xi=\left(\frac{2 N}{k}-1\right) \xi .
$$

Thus $\|a\| \geq \frac{2 N}{k}-1$.

Proposition 7.6. Let $H=\bigoplus_{i=1}^{N} H_{i}$ be a Hilbert space direct sum, $x \in \mathcal{B}(H)$ and $\mathcal{C}$ be a simplicial complex on $\{1,2, \ldots, N\}$. We write $x=\left(x_{i, j}\right)_{i, j=1}^{N}$ as a block $N \times N$ matrix with entries $x_{i, j} \in \mathcal{B}\left(H_{j}, H_{i}\right)$, and for $E \subseteq\{1,2, \ldots, N\}$, let $x_{E}=\left(\chi_{E}(i) \chi_{E}(j) x_{i, j}\right)_{i, j=1}^{N}$ (where $\chi_{E}$ means the characteristic function of $E$ ). Then

$$
\|x\| \leq L_{\mathbb{C}}(\mathcal{C}) \max _{E \in \mathcal{C}}\left\|x_{E}\right\|
$$

Proof. We assume for convenience that $\max _{E \in \mathcal{C}}\left\|x_{E}\right\|=1$. For unit vectors $\xi, \eta \in$ $H$, we aim to estimate $|\langle x \eta, \xi\rangle|$ by $L_{\mathbb{C}}(\mathcal{C})$. Let $\xi=\sum_{i=1}^{N} \xi_{i}, \eta=\sum_{i=1}^{N} \eta_{i}$ for $\xi_{i}, \eta_{i} \in H_{i}(1 \leq i \leq N)$.

Define $\xi_{i}^{\prime}=\xi_{i} /\left\|\xi_{i}\right\|$ (unless $\xi_{i}=0$, in which case we take $\xi_{i}^{\prime} \in H_{i}$ to be any unit vector). Similarly define $\eta_{i}^{\prime}$ to be the unit vector in the direction of $\eta_{i}$. Then we 
have orthogonal vectors $\left\{\xi_{i}^{\prime}: 1 \leq i \leq N\right\}$ and $\left\{\eta_{i}^{\prime}: 1 \leq i \leq N\right\}, \eta=\sum_{i=1}^{N}\left\|\eta_{i}\right\| \eta_{i}^{\prime}$, $1=\|\eta\|^{2}=\sum_{i=1}^{N}\left\|\eta_{i}\right\|^{2}$, and similarly for $\xi$. For any scalars $\alpha=\left(\alpha_{i}\right)_{i=1}^{N} \in \ell_{N}^{2}$ we have $\left\|\sum_{i=1}^{N} \alpha_{i} \eta_{i}^{\prime}\right\|^{2}=\sum_{i=1}^{N}\left|\alpha_{i}\right|^{2}$. Let $a_{i, j}=\left\langle x_{i, j} \eta_{j}^{\prime}, \xi_{i}^{\prime}\right\rangle$ and take $a=\left(a_{i, j}\right)_{i, j=1}^{N} \in$ $M_{N}$.

Now if $E \in \mathcal{C}$ and $\alpha_{E}=\left(\alpha_{i}\right)_{i \in E} \in \ell_{E}^{2}, \beta_{E}=\left(\beta_{i}\right)_{i \in E} \in \ell_{E}^{2}$, then

$$
\begin{aligned}
\left|\left\langle a_{E} \alpha_{E}, \beta_{E}\right\rangle\right| & =\left|\left\langle\sum_{i, j \in E} a_{i, j} \alpha_{j} \beta_{i}\right\rangle\right| \\
& =\left|\left\langle x\left(\sum_{i \in E} \alpha_{i} \eta_{i}^{\prime}\right), \sum_{i \in E} \beta_{i} \xi_{i}^{\prime}\right\rangle\right| \\
& \leq\left(\sum_{i \in E}\left|\alpha_{i}\right|^{2} \sum_{j \in E}\left|\beta_{j}\right|^{2}\right)^{1 / 2}
\end{aligned}
$$

(using $\left\|x_{E}\right\| \leq 1$ ). Hence $\left\|a_{E}\right\| \leq 1$ for $E \in \mathcal{C}$, and so $\|a\| \leq L_{\mathbb{C}}(\mathcal{C})$.

Hence

$$
|\langle x \eta, \xi\rangle|=\left|\sum_{i, j=1}^{N} a_{i, j}\left\|\eta_{j}\right\|\left\|\xi_{i}\right\|\right|=\left|\left\langle a\left(\left\|\eta_{j}\right\|\right)_{j=1}^{N},\left(\left\|\xi_{i}\right\|\right)_{i=1}^{N}\right\rangle\right| \leq\|a\| \leq L_{\mathbb{C}}(\mathcal{C}) .
$$

Proposition 7.7. For $\mathcal{C}$ a simplicial complex on $\{1,2, \ldots, N\}$,

$$
L^{\prime}(A(\mathcal{C})) \leq L_{\mathbb{C}}(\mathcal{C})
$$

Proof. From Lemma 4.7 we have an expression for $L\left(M_{n}(A(\mathcal{C}))\right)$, upon which we base our argument. We can consider $\mathbb{C}^{E} \subset \mathbb{C}^{N}$ as the elements of $\mathbb{C}^{N}$ supported on $E$, which is a ${ }^{*}$-isomorphic (non-unital) embedding of commutative $C^{*}$-algebras. We also consider $\mathbb{C}^{N} \subset M_{N}$ via the diagonal, so that if $e_{i, i} \in M_{N}$ denotes the diagonal matrix with 1 in the $(i, i)$ entry, then $f=\left(f_{1}, f_{2}, \ldots, f_{n}\right) \in \mathbb{C}^{N}$ corresponds to $\sum_{i=1}^{N} f_{i} e_{i, i}$ and $g=\left(g_{i}\right)_{i \in E} \in \mathbb{C}^{E}$ corresponds to $\sum_{i \in E} g_{i} e_{i, i}$. Subject to these identifications we can write $P_{E}(f)$ as the product $p_{E} f=f p_{E}$, where $p_{E}=\sum_{i \in E} e_{i, i} \in M_{N}$ is a projection.

For $a \in \mathbb{C}^{N} \otimes M_{n}=M_{n}\left(\mathbb{C}^{N}\right)$, we consider $a$ as an element $a \in M_{N} \otimes M_{n}$ and then identify $P_{E}^{(n)}(a)$ (notation as in Lemma 4.7) with $\left(p_{E} \otimes I_{n}\right) a=a\left(p_{E} \otimes I_{n}\right)$.

Fix $v=\sum_{j=1}^{\ell} a_{j} \otimes b_{j} \in M_{n}\left(\mathbb{C}^{N}\right) \otimes M_{n}\left(\mathbb{C}^{N}\right)$, with the aim of showing $\|v\|_{h} \leq$ $L_{\mathbb{C}}(\mathcal{C}) \max _{E \in \mathcal{C}}\left\|v_{E}\right\|_{h}$. As before, we make use of the fact that $\|v\|_{h}$ is the cb norm of the elementary operator $T=\theta(v)$ on $M_{N} \otimes M_{n}$, via injectivity of $\|\cdot\|_{h}$ and Haagerup's theorem. We can also see that $\left\|v_{E}\right\|_{h}=\left\|T_{E}\right\|_{c b}$, where $T_{E}: M_{N} \otimes M_{n} \rightarrow$ $M_{N} \otimes M_{n}$ is

$$
T_{E}(x)=\sum_{j=1}^{\ell}\left(\left(p_{E} \otimes I_{n}\right) a_{j}\right) x\left(\left(p_{E} \otimes I_{n}\right) b_{j}\right) .
$$


We also know (from [19], Proposition 8.11]) that $\|T\|_{c b}=\left\|T^{(m)}\right\|$ with $m=n N$, where $T^{(m)}$ is the elementary operator on $M_{n}\left(M_{N}\right) \otimes M_{m} \cong M_{n N m}$ given by

$$
T^{(m)}(x)=\left(T \otimes \operatorname{id}_{m}\right)(x)=\sum_{j=1}^{\ell}\left(a_{j} \otimes I_{m}\right) x\left(b_{j} \otimes I_{m}\right) .
$$

We consider $x \in M_{n N m}$ of norm 1 and seek to estimate $\|y\|$ for $y=T^{(m)}(x)$.

We write

$$
x_{E}=\left(p_{E} \otimes I_{n} \otimes I_{m}\right) x\left(p_{E} \otimes I_{n} \otimes I_{m}\right)
$$

and

$$
y_{E}=\left(p_{E} \otimes I_{n} \otimes I_{m}\right) y\left(p_{E} \otimes I_{n} \otimes I_{m}\right) .
$$

It is important to observe that the notation $y_{E}$ accords with the notation of Proposition 7.6 if we take $H_{i}$ to be the range of $p_{\{i\}} \otimes I_{n} \otimes I_{m}(1 \leq i \leq N)$. Note also that $\left\|x_{E}\right\| \leq\|x\| \leq 1$. We can compute, using the shorthand $\tilde{p}_{E}=p_{E} \otimes I_{n} \otimes I_{m}$,

$$
\begin{aligned}
y_{E} & =\tilde{p}_{E}\left(\sum_{j=1}^{\ell}\left(a_{j} \otimes I_{m}\right) x\left(b_{j} \otimes I_{m}\right)\right) \tilde{p}_{E} \\
& =\sum_{j=1}^{\ell}\left(\left(p_{E} \otimes I_{n}\right) a_{j} \otimes I_{m}\right) \tilde{p}_{E} x \tilde{p}_{E}\left(\left(p_{E} \otimes I_{n}\right) b_{j} \otimes I_{m}\right) \\
& =T_{E}^{(m)}\left(x_{E}\right) .
\end{aligned}
$$

We deduce $\left\|y_{E}\right\| \leq\left\|T_{E}\right\|_{c b}\left\|x_{E}\right\| \leq\left\|v_{E}\right\|_{h}$. Thus $\|y\| \leq L_{\mathbb{C}}(\mathcal{C}) \max _{E \in \mathcal{C}}\left\|v_{E}\right\|_{h}$ by Proposition 7.6 .

As $x$ was arbitrary of unit norm, we have

$$
\|v\|_{h}=\|T\|_{c b}=\left\|T^{(m)}\right\| \leq L_{\mathbb{C}}(\mathcal{C}) \max _{E \in \mathcal{C}}\left\|v_{E}\right\|_{h} .
$$

Thus $L\left(M_{n}(A(\mathcal{C}))\right) \leq L_{\mathbb{C}}(\mathcal{C})$ (by Lemma 4.7), and so

$$
L^{\prime}(A(\mathcal{C}))=\sup _{n} L\left(M_{n}(A(\mathcal{C}))\right) \leq L_{\mathbb{C}}(\mathcal{C})
$$

Corollary 7.8. For $N>k \geq 2, L^{\prime}\left(A\left(\mathcal{C}_{N, k}\right)\right) \leq \frac{2 N}{k}-1$.

Proof. From Proposition 7.7 and Proposition $7.5 L^{\prime}\left(A\left(\mathcal{C}_{N, k}\right)\right) \leq L_{\mathbb{C}}\left(\mathcal{C}_{N, k}\right)=$ $\frac{2 N}{k}-1$.

Proposition 7.9. For $N>k \geq 2, L^{\prime}\left(A\left(\mathcal{C}_{N, k}\right)\right) \geq L\left(M_{N}\left(A\left(\mathcal{C}_{N, k}\right)\right)\right) \geq(N-1) /(k-1)$.

Proof. By Lemma 4.7 it is sufficient to exhibit a tensor $v \in M_{N}\left(\mathbb{C}^{N}\right) \otimes M_{N}\left(\mathbb{C}^{N}\right)$ such that $\|v\|_{h} \geq N-1$ and $\sup \left\|v_{E}\right\|_{h} \leq k-1$ (with the supremum over $E \in \mathcal{C}_{N, k}$ ).

Let $e_{i, j} \in M_{N}$ be the matrix with 1 in the $(i, j)$ position and zeros elsewhere. We consider $\mathbb{C}^{N}$ as being embedded in $M_{N}$ via the diagonal $\left\{e_{i, i}: 1 \leq i \leq N\right\}$, and then $M_{N}\left(\mathbb{C}^{N}\right)=\mathbb{C}^{N} \otimes M_{N} \subseteq M_{N} \otimes M_{N}$. Let $v=\sum_{1 \leq i, j \leq N, i \neq j}\left(e_{i, i} \otimes e_{1, j}\right) \otimes\left(e_{j, j} \otimes e_{i, 1}\right)$.

We consider the elementary operator $T=\theta(v) \in \mathcal{B}\left(M_{N} \otimes M_{N}\right)=\mathcal{B}\left(M_{N^{2}}\right)$. Consider the operator $x=\sum_{1 \leq i, j \leq N} e_{j, i} \otimes e_{i, j} \in M_{N} \otimes M_{N}$ :

$$
x^{*} x=\sum_{i, j, r, s} e_{i, j} e_{s, r} \otimes e_{j, i} e_{r, s}=\sum_{r, s} e_{r, s} e_{s, r} \otimes e_{s, r} e_{r, s}=\sum_{r, s} e_{r, r} \otimes e_{s, s}=I_{N} \otimes I_{N},
$$


and so $\|x\|=1$. We compute

$$
\begin{aligned}
T x & =\sum_{i \neq j}\left(e_{i, i} \otimes e_{1, j}\right) x\left(e_{j, j} \otimes e_{i, 1}\right) \\
& =\sum_{i \neq j, r, s}\left(e_{i, i} \otimes e_{1, j}\right)\left(e_{s, r} \otimes e_{r, s}\right)\left(e_{j, j} \otimes e_{i, 1}\right) \\
& =\sum_{i \neq j, r, s} e_{i, i} e_{s, r} e_{j, j} \otimes e_{1, j} e_{r, s} e_{i, 1} \\
& =\sum_{i \neq j} e_{i, i} e_{i, j} e_{j, j} \otimes e_{1, j} e_{j, i} e_{i, 1}=\sum_{i \neq j} e_{i, j} \otimes e_{1,1} .
\end{aligned}
$$

The matrix $\sum_{i \neq j} e_{i, j} \in M_{N}$ has eigenvalue $N-1$ on the vector $(1,1, \ldots, 1) \in \mathbb{C}^{N}$. It follows that $\|T x\| \geq N-1$. Thus $\|T\| \geq N-1$, and so $\|v\|_{h}=\|T\|_{c b} \geq N-1$.

When we look at

$$
v_{E}=\left(P_{E}^{(N)} \otimes P_{E}^{(N)}\right)(v)=\left(\left(P_{E} \otimes \mathrm{id}_{N}\right) \otimes\left(P_{E} \otimes \mathrm{id}_{N}\right)\right)(v)
$$

for $E \in \mathcal{C}_{N, k}$, we see that

$$
v_{E}=\sum_{i, j \in E, i \neq j}\left(e_{i, i} \otimes e_{1, j}\right) \otimes\left(e_{j, j} \otimes e_{i, 1}\right),
$$

and we verify that $\left\|v_{E}\right\|_{h} \leq k-1$, as follows. It suffices to consider $E$ of the maximal possible cardinality $k$ :

$$
\begin{aligned}
& \left\|v_{E}\right\|_{h}^{2} \\
& \leq\left\|\sum_{i, j \in E, i \neq j}\left(e_{i, i} \otimes e_{1, j}\right)\left(e_{i, i} \otimes e_{1, j}\right)^{*}\right\|\left\|_{i, j \in E, i \neq j}\left(e_{j, j} \otimes e_{i, 1}\right)^{*}\left(e_{j, j} \otimes e_{i, 1}\right)\right\| \\
& =\left\|\sum_{i, j \in E, i \neq j}\left(e_{i, i} \otimes e_{1,1}\right)\right\| \sum_{i, j \in E, i \neq j}\left(e_{j, j} \otimes e_{1,1}\right) \| \\
& =\left\|(k-1) I_{E} \otimes e_{1,1}\right\|\left\|(k-1) I_{E} \otimes e_{1,1}\right\|=(k-1)^{2}
\end{aligned}
$$

(where $I_{E}=\sum_{i \in E} e_{i, i}$ ).

Theorem 7.10. For $N \geq 3, L^{\prime}\left(A\left(\mathcal{C}_{N, 2}\right)\right)=N-1$.

Proof. The result follows from Corollary 7.8 and Proposition 7.9 .

Corollary 7.11. Let $A$ be a $C^{*}$-algebra and $n \geq 2$. Suppose that each $G \in$ $\operatorname{Glimm}(A)$ is 2-primal and satisfies $|\operatorname{Prim}(A / G)| \leq n$. Then $L^{\prime}(A) \leq n-1$.

Proof. From the hypotheses each simplicial complex $\mathcal{C}$ linked to the ideal structure of $A$ will have $N=1$ or $\mathcal{C}_{N, 2} \subseteq \mathcal{C}$. As in Notation 4.6. $L^{\prime}(A(\mathcal{C}))=1$ if $N=1$ (or $N=2$ ). If $N>1$, we then have $L^{\prime}(A(\mathcal{C})) \leq L^{\prime}\left(A\left(\mathcal{C}_{N, 2}\right)\right)=N-1 \leq n-1$ (using Remark 4.8 (c). From Theorem 6.1 we get $L^{\prime}(A) \leq n-1$.

Examples 7.12. (i) The special case $A\left(\mathcal{C}_{N, k}\right)$ with $k=N-1$ was considered in [10. A note in [10, Remark 3] implies that

$$
L\left(A\left(\mathcal{C}_{N, N-1}\right)\right) \geq 1 /\left(1-\frac{1}{(N-1)^{2}}\right)>1 \quad(N \geq 3) .
$$


From Corollary 7.8 we have

$$
L\left(A\left(\mathcal{C}_{N, N-1}\right)\right) \leq L^{\prime}\left(A\left(\mathcal{C}_{N, N-1}\right)\right) \leq 1+\frac{2}{N-1} .
$$

Thus, among the values $L^{\prime}\left(A\left(\mathcal{C}_{N, N-1}\right)\right)$, there are infinitely many distinct values tending to 1 (from above). The same is true of the values $L\left(A\left(\mathcal{C}_{N, N-1}\right)\right)$.

The complexes $\mathcal{C}_{N, N-1}$ are extremal in a certain sense. If $\mathcal{C}$ is any simplicial complex on $\{1,2, \ldots, N\}$ other than the power set, then $L\left(A\left(\mathcal{C}_{N, N-1}\right)\right) \leq L(A(\mathcal{C}))$ by Remark 4.8 (c). If a $C^{*}$-algebra $A$ contains a Glimm ideal $G$ that fails to be $N$-primal (for some $N \geq 3$ ), we have $L\left(A\left(\mathcal{C}_{N, N-1}\right)\right) \leq L(A)$ and $L^{\prime}\left(A\left(\mathcal{C}_{N, N-1}\right)\right) \leq$ $L^{\prime}(A)$. This follows from Theorem 5.1 if $G$ is 2-primal. If $G$ is not 2-primal, then $L^{\prime}(A) \geq L(A)=\infty$ by Remark 4.2

(ii) If $N>k \geq 2 N / 3$ (and $N \geq 3$ ), then $L^{\prime}\left(A\left(\mathcal{C}_{N, k}\right)\right) \leq 2$ by Corollary 7.8 .

(iii) Recalling $L\left(A\left(\mathcal{C}_{N, 2}\right)\right) \leq \sqrt{N}$ from Remarks 7.1 (i), we have $L\left(A\left(\mathcal{C}_{N, 2}\right)\right)<$ $L^{\prime}\left(A\left(\mathcal{C}_{N, 2}\right)\right)=N-1($ for $N>2)$.

We now give examples for which $L(A) \leq 2<L^{\prime}(A)=\infty$.

Proposition 7.13. Let $\mathcal{C}$ be the simplicial complex

$$
\mathcal{C}=\mathcal{B}_{N, k-1}=\{E \subset\{1,2, \ldots, N\}: 1 \notin E \text { or }|E \backslash\{1\}| \leq k-1\}
$$

and $A=A(\mathcal{C})$ (where $N>k \geq 2$ ). Then $L^{\prime}(A) \geq L\left(M_{N}(A)\right) \geq \sqrt{\frac{N-1}{k-1}}$ while $L(A) \leq 2$.

Proof. The stated lower bound for $L\left(M_{N}(A)\right)$ follows from the argument in the proof of Proposition 7.9 with $v$ replaced by $v=\sum_{i=2}^{N}\left(e_{i, i} \otimes e_{1,1}\right) \otimes\left(e_{1,1} \otimes e_{i, 1}\right)$.

By Lemma 4.7 and Remarks 4.8 (a), establishing the upper bound for $L(A(\mathcal{C}))$ can be reduced to a question about Schur multipliers. Assume $T$ is a Schur multiplier on $M_{N}$ given by $T x=\left(\gamma_{i j} x_{i j}\right)_{i, j=1}^{N}$ and assume that the restricted Schur multipliers $T_{E}$ on $M_{E}$ have norms bounded by 1 for each $E \in \mathcal{B}_{N, k-1}$. In particular for $E_{0}=\{2,3, \ldots, N\}$ we have $\left\|T_{E_{0}}\right\| \leq 1$ and for $E=\{1, i\}$ with $2 \leq i \leq N$, we have $\left\|T_{E}\right\| \leq 1$ which implies $\left|\gamma_{11}\right| \leq 1,\left|\gamma_{1 i}\right| \leq 1$ and $\left|\gamma_{i 1}\right| \leq 1$. Expressing $T x$ as

$$
\left(\begin{array}{cccc}
\gamma_{11} x_{11} & 0 & \cdots & 0 \\
0 & \gamma_{22} x_{22} & \cdots & \gamma_{2 N} x_{2 N} \\
\vdots & \vdots & \ddots & \vdots \\
0 & \gamma_{N 2} x_{N 2} & \cdots & \gamma_{N N} x_{N N}
\end{array}\right)+\left(\begin{array}{cccc}
0 & \gamma_{12} x_{12} & \cdots & \gamma_{1 n} x_{1 n} \\
\gamma_{21} x_{21} & 0 & \cdots & 0 \\
\vdots & \vdots & \ddots & \vdots \\
\gamma_{N 1} x_{N 1} & 0 & \cdots & 0
\end{array}\right)
$$

and using the fact that the norm of the second summand is the maximum of the $\ell^{2}$ norms of the first row and first column, it follows that $\|T x\| \leq 2\|x\|$.

Regarding Proposition 7.13, note that if $(N-1) /(k-1)>4$, then $L^{\prime}(A)>L(A)$.

Corollary 7.14. With the notation of Proposition 7.13, let $A$ be the $c_{0}$ direct sum of $A\left(\mathcal{B}_{N, 1}\right)(N=3,4, \ldots)$. Then $L(A) \leq 2$ while $L^{\prime}(A)=\infty$.

Proof. By Corollary 4.5 and Proposition 7.13, $L(A) \leq 2$ and $L^{\prime}(A)=\infty$.

In fact it is also true that the unitisation $A_{1}$ of the algebra $A$ in Corollary 7.14 satisfies $L^{\prime}\left(A_{1}\right)=\infty$. We can establish a variant of Corollary 4.5 for the unitisation of the direct sum, with a somewhat similar proof, and this allows us to show $L^{\prime}\left(A_{1}\right)=\infty$. 
If we recall from Corollary 6.3 that $L^{\prime}(A)=L(A \otimes \mathcal{K}(H))$, then we see that $L(A \otimes \mathcal{K}(H))=\infty$ but $L(A) \leq 2$, even though $A$ and $A \otimes \mathcal{K}(H)$ have homeomorphic primitive ideals spaces.

Proposition 7.15. Let $A$ be a $C^{*}$-algebra such that each Glimm ideal is 2-primal. Assume $G \in \operatorname{Glimm}(A)$ and there exists $3 \leq k \leq N, P_{1}, P_{2}, \ldots, P_{N} \in \operatorname{Prim}(A / G)$ so that

- $P_{i} \not \subset P_{j}$ for $i \neq j$; and

- no intersection of $k$ of $P_{1}, P_{2}, \ldots, P_{N}$ is primal.

Then

(i) $L(A) \geq \sqrt{N /(k-1)}$, and

(ii) $L^{\prime}(A) \geq L\left(M_{N}(A)\right) \geq(N-1) /(k-2)$.

Proof. The relevant simplicial complex $\mathcal{C}=\left\{E \subseteq\{1,2, \ldots, N\}: \bigcap_{i \in E} P_{i} \in\right.$ $\operatorname{Primal}(A)\}$ is contained in $\mathcal{C}_{N, k-1}$. So by Theorem 5.1. Remarks 4.8 (c) and Examples 7.1 (i) we have $L(A) \geq L(A(\mathcal{C})) \geq L\left(A\left(\mathcal{C}_{N, k-1}\right)\right) \geq \sqrt{N /(k-1)}$.

Similarly $L\left(M_{N}(A)\right) \geq L\left(M_{N}\left(A\left(\mathcal{C}_{N, k-1}\right)\right)\right) \geq(N-1) /(k-2)$ by Proposition 7.9 ,

Using Proposition 7.15, we can now determine $L(A)$ and $L^{\prime}(A)$ for certain group $C^{*}$-algebras.

Example 7.16. (i) Let $n \geq 3$ be an odd integer and let $G=W_{n}$ be the "universal" simply connected, two-step nilpotent Lie group studied in [8, section 2]. Let $\mathfrak{z}_{n}$ be the centre of the Lie algebra of $G$. For $f \in \mathfrak{z}_{n}^{\perp}$, the annihilator of $\mathfrak{z}_{n}$ in the dual of the Lie algebra, let $\pi_{f}$ be the corresponding irreducible representation of $C^{*}(G)$. It follows from [8, Corollary 2.8 (i)] that every Glimm ideal of $C^{*}(G)$ is 2-primal (and hence $\theta_{Z}$ is injective for $A=C^{*}(G)$ by Theorem 3.8 (i)). We shall construct a sequence $\left(f_{j}\right)_{j \geq 1}$ in $\mathfrak{z}_{n}^{\perp}$ such that, for each $N \geq n+1$, no $n+1$ of $f_{1}, \ldots, f_{N}$ lie in an affine hyperplane in $\mathfrak{z}_{n}^{\perp}$. It will then follow from 8 , Theorem 2.7 (ii)] that, for each $N \geq n+1$, no $n+1$ of $\operatorname{ker} \pi_{f_{1}}, \ldots, \operatorname{ker} \pi_{f_{N}}$ have primal intersection. Hence, by Proposition 7.15, $L\left(C^{*}(G)\right) \geq \sqrt{N / n}$ for all $N \geq n+1$, and so $L^{\prime}\left(C^{*}(G)\right)=L\left(C^{*}(G)\right)=\infty$.

To construct the sequence $\left(f_{j}\right)_{j \geq 1}$, we may begin by taking $f_{1}=0$ and $\left\{f_{2}, f_{3}, \ldots\right.$, $\left.f_{n+1}\right\}$ to be any basis for $\mathfrak{z}_{n}^{\perp}$. Now suppose that $N \geq n+1$ and that $f_{1}, \ldots, f_{N}$ have been constructed with the required property. Then, in choosing $f_{N+1}$, we simply have to avoid $\left(\begin{array}{l}N \\ n\end{array}\right)$ affine hyperplanes in $\mathfrak{z}_{n}^{\perp}$.

(ii) Let $G_{N}(N \geq 3)$ be the 'thread-like' nilpotent Lie groups (see, for example, $\left[12,6\right.$, 8]). The group $G_{3}$ is the continuous Heisenberg group. The group $G_{4}$ also has the property that every Glimm ideal of its $C^{*}$-algebra is primal 6]. However, this property fails for $G_{N}$ with $N \geq 5$ (see [6] and [8] Theorem 3.1]). For $N \geq 5$, it follows from [12] (see also [8, Theorem 3.9 (iii)] and [9, Theorem 3.2]) that there are uncountably many primitive ideals of $C^{*}\left(G_{N}\right)$ which have the property that they belong to the same Glimm class as the $C^{*}$-kernel of the trivial representation and no $N-1$ of them have primal intersection. It follows from Proposition 7.15 that $L^{\prime}\left(C^{*}\left(G_{N}\right)\right)=L\left(C^{*}\left(G_{N}\right)\right)=\infty$. This is already apparent in the case where $N>4$ and $N \equiv 0 \bmod 4$ because the relation of inseparability on $\widehat{G_{N}}$ is not transitive [8, Corollary 3.10] and so $C^{*}\left(G_{N}\right)$ has a Glimm ideal that is not 2-primal. On the 
other hand, if $N$ is odd $(N \geq 5)$, then it follows from [8, Theorem 3.9] and the discussion for $G_{7}$ on [8, p. 289] that every Glimm ideal of $C^{*}\left(G_{N}\right)$ is 2-primal. It is an interesting open question as to whether every Glimm ideal of $C^{*}\left(G_{N}\right)$ is 2-primal in the case where $N \equiv 2 \bmod 4$.

\section{REFERENCES}

[1] S. D. Allen, A. M. Sinclair and R. R. Smith, The ideal structure of the Haagerup tensor product of $C^{*}$-algebras, J. Reine Angew. Math. 442 (1993) 111-148. MR1234838 (94k:46107)

[2] C. A. Akemann and G. K. Pedersen, Ideal perturbations of elements in $C^{*}$-algebras, Math. Scand. 41 (1977) 117-139. MR0473848 (57:13507)

[3] P. Ara and M. Mathieu, Local multipliers of $C^{*}$-algebras, Springer-Verlag London, Ltd., London 2003. MR1940428 (2004b:46071)

[4] R. J. Archbold, Topologies for primal ideals, J. London Math. Soc. (2) 36 (1987) 524-542. MR.918643 (89h:46076)

[5] R. J. Archbold and C. J. K. Batty, On factorial states of operator algebras III, J. Operator Theory 15 (1986) 53-81. MR816234 (87h:46126)

[6] R. J. Archbold and E. Kaniuth, Simply connected nilpotent Lie groups with quasi-standard $C^{*}$-algebras, Proc. Amer. Math. Soc. 125 (1997) 2733-2742. MR1389503 (97j:22009)

[7] R. J. Archbold, E. Kaniuth, G. Schlichting and D. W. B. Somerset, Ideal spaces of the Haagerup tensor product of $C^{*}$-algebras, Int. J. Math. 8 (1997) 1-29. MR:1433199 (97m:46089)

[8] R. J. Archbold, E. Kaniuth, G. Schlichting and D. W. B. Somerset, On the topology of the dual of a nilpotent Lie group, Math. Proc. Camb. Phil. Soc. 125 (1999) 269-293. MR.1643794 (2000b:22010)

[9] R. J. Archbold, J. Ludwig and G. Schlichting, Limit sets and strengths of convergence for sequences in the duals of thread-like Lie groups, Math. Zeit. 255 (2007) 245-282. MR2262731 $(2007 \mathrm{j}: 22017)$

[10] R. J. Archbold, D. W. B. Somerset and R. M. Timoney, On the central Haagerup tensor product and completely bounded mappings of a $C^{*}$-algebra, J. Funct. Anal. 226 (2005) 406428. MR2160102 (2006d:46066)

[11] C. J. K. Batty and R. J. Archbold, On factorial states of operator algebras II, J. Operator Theory 13 (1985) 131-142. MR768307/(86f:46065)

[12] J. Boidol, J. Ludwig and D. Müller, On infinitely small orbits, Studia Math. 88 (1988) 1-11. MR932001 (89f:22009)

[13] A. Chatterjee and R. R. Smith, The central Haagerup tensor product and maps between von Neumann algebras, J. Funct. Anal. 112 (1993) 97-120. MR1207938 (94c:46120)

[14] J. Dauns, The primitive ideal space of a $C^{*}$-algebra, Canadian J. Math. 26 (1974) 42-49. MR0336356 (49:1131)

[15] J. Dauns and K.-H. Hofmann, Representation of rings by sections, Memoirs of the American Mathematical Society, No. 83, American Mathematical Society, Providence 1968. MR 0247487 (40:752)

[16] J. Dixmier, Les $C^{*}$-algèbres et leurs représentations, Gauthier-Villars, Paris 1964. MR0171173 (30:1404)

[17] A. Guichardet, Tensor products of $C^{*}$-algebras, (Part I, Finite tensor products), Aarhus University, Math. Institute, Lecture Notes Series No. 12, 1969.

[18] E. Kaniuth, G. Schlichting and K. F. Taylor, Minimal primal and Glimm ideal spaces of group $C^{*}$-algebras, J. Funct. Anal. 130 (1995) 43-76. MR1331977(96g:46045)

[19] V. Paulsen, Completely bounded maps and operator algebras, Cambridge Univ. Press, Cambridge 2002. MR1976867 (2004c:46118)

[20] I. Raeburn and D. P. Williams, Morita Equivalence and Continuous-Trace $C *$-Algebras, Mathematical Surveys and Monographs Volume 60, American Mathematical Society, Providence 1998. MR 1634408 (2000c:46108)

[21] D. W. B. Somerset, Inner derivations and primal ideals of $C^{*}$-algebras, J. London Math. Soc. (2) 50 (1994) 568-580. MR1299458 (95h:46107)

[22] D. W. B. Somerset, The central Haagerup tensor product of a $C^{*}$-algebra, J. Operator Theory 39 (1998) 113-121. MR.1610306 (99e:46073) 
[23] R. M. Timoney, Some formulae for norms of elementary operators, J. Operator Theory 57 (2007) 121-145. MR:2301939 (2007m:47088)

[24] J. Tomiyama, Topological representations of $C^{*}$-algebras, Tôhoku Math. J. (2) 14 (1962) 187-204. MR0143053(26:619)

Department of Mathematical Sciences, University of Aberdeen, King's College, Aberdeen AB24 3UE, Scotland, United Kingdom

E-mail address: r.archbold@maths.abdn.ac.uk

Department of Mathematical Sciences, University of Aberdeen, King's College, Aberdeen AB24 3UE, Scotland, United Kingdom

E-mail address: somerset@quidinish.fsnet.co.uk

School of Mathematics, Trinity College, Dublin 2, Ireland

E-mail address: richardt@maths.tcd.ie 